Article

\title{
Logic-based modeling and virtual drug screening for the prediction of novel therapeutic targets and combination regimens against E2F1-driven melanoma progression
}

\author{
Nivedita Singh ${ }^{1}$, Faiz M Khan ${ }^{2}$, Lakshmi Bala ${ }^{1}$, Julio Vera ${ }^{4}$, Olaf Wolkenhauer ${ }^{2,5,6}$, Brigitte Pützer ${ }^{3}$, \\ Stella Logotheti ${ }^{3}$, Shailendra K. Gupta ${ }^{2,6^{*}}$ \\ ${ }^{1}$ Department of Biochemistry, BBDCODS, BBD University, Lucknow, 226028, Uttar Pradesh, India \\ ${ }^{2}$ Department of Systems Biology and Bioinformatics, University of Rostock, Rostock 18051, Germany \\ ${ }^{3}$ Institute of Experimental Gene Therapy and Cancer Research, Rostock University Medical Center, 18057 \\ Rostock, Germany \\ ${ }^{4}$ Department of Dermatology, Laboratory of Systems Tumor Immunology, Erlangen University Hospital and \\ FAU University of Erlangen-Nuremberg, 91054 Erlangen, Germany \\ ${ }^{5}$ Leibniz Institute for Food Systems Biology at the Technical University of Munich, Munich, Germany \\ ${ }^{6}$ Chhattisgarh Swami Vivekanand Technical University, Bhilai, Chhattisgarh 491107, India \\ * Correspondance : shailendra.gupta@uni-rostock.de; Tel.: +49 3814987685
}

Simple Summary: The standard-of-care treatments of melanoma induce rapid responses but are linked to the development of resistance and/or increased risk of side effects for toxicities, highlighting an urgent need for novel targeted therapies with favorable efficacy and safety profiles. Given that melanoma progression is mediated by activation of a prometastatic network, controlled by the transcription factor E2F1, drugs that perturb this network could potentially prevent metastasis. However, this network is highly interconnected and forms complex loops, making it difficult to identify druggable targets. Systems pharmacology facilitates the characterization of this network and improves the identification of drugs acting against it. We constructed the E2F1-controlled network underlying melanoma progression and, using a systems approach, we predicted which proteins are likely to prevent metastasis, if targeted. Then, based on the structures of AKT and MDM2, we virtually screened compounds to identify leads that can bind to these proteins and inhibit their activity, taking also into account their toxicity profiles. Overall, we identified specific AKT and MDM2 inhibitors which could constitute efficient and safe therapeutic options for aggressive melanoma, either alone or in combination.

\footnotetext{
Abstract: Skin melanoma presents increasing prevalence and poor outcomes. Progression to aggressive stages is characterized by overexpression of the transcription factor E2F1 and activation of downstream prometastatic gene regulatory networks (GRNs). Appropriate therapeutic manipulation of the E2F1-governed GRNs holds potential to prevent metastasis, however these networks entail complex feedback and feedforward regulatory motifs among various regulatory layers, which make it difficult to identify druggable components. To this end, computational approaches such as mathematical modeling and virtual screening are important tools to unveil the dynamics of these signaling networks and identify critical components that could be further
} 
explored as therapeutic targets. Herein, we integrated a well-established E2F1-mediated epithelialmesenchymal transition (EMT) map with transcriptomics data from E2F1-expressing melanoma cells to reconstruct a core regulatory network underlying aggressive melanoma. Using logic-based in silico perturbation experiments of a core regulatory network, we identified that simultaneous perturbation of AKT1 and MDM2 drastically reduces EMT in metastatic melanoma. Using the structures of the two protein signatures along with virtual screening of lead-like compound library available in ZINC12 database, we identified a number of lead compounds that efficiently inhibit AKT1 and MDM2 without eliciting toxicities. We propose that these compounds could be taken into account in the design of novel therapeutic strategies for the management of aggressive melanoma.

Keywords: Melanoma; Systems pharmacology; Boolean model; Small molecule inhibitors; Virtual screening, ADME, E2F1

\section{Introduction}

Cutaneous melanoma arises from melanocytes and represents the deadliest form of skin cancer, with increasing prevalence. Once it becomes metastatic, the prognosis is very unfavorable. Melanoma formation is driven by mutations in the BRAF and NRAS oncogenes [1]. However, these oncogenic aberrations are early events in melanomagenesis that alone do not seem to be sufficient to drive metastasis [2]. Over the past years, we [3-5] and others [6] have demonstrated that in addition to these driver events, melanoma progression is catalyzed by the abundant expression of E2F1, a member of the E2F transcription factor family. Although this transcription factor activates tumor-suppressive pathways at early oncogenesis, upon disease progression unbalanced E2F1 activity is rewired to deregulated cancer networks that underlie hallmarks of metastatic progression, such as resistance to apoptosis, chemoresistance [3,7], neoangiogenesis [8], extravasation [9], epithelial-mesenchymal transition (EMT) [10,11], metabolic reprogramming [12], and genomic instability [13]. By integrating logic-based network modeling and gene expression profiles of cancer cell lines from E2F1driven tumors and patient cohorts displaying cancer aggressiveness, we identified tumor-type specific receptor signatures associated to EMT, where the combined action of highly expressed E2F1, TGFBR1 and FGFR1 triggers the most invasive phenotype [11,14]. Several other protein coding genes (PCGs), miRNA genes and lncRNA genes have been identified as constituents of E2F1-activated prometastatic gene regulatory networks (GRNs) [11-13,15,16]. Within the E2F1-governed GRNs, non-linear feedback and feedforward regulatory motifs are formed among various regulatory network layers, entailing protein-coding and non-coding RNA genes $[10-13,15,16]$. Such regulatory motifs, which are commonly encountered in cancer networks $[17,18]$, induce a whole range of dynamic behaviors, thereby evading the use of conventional data analysis methods [19]. As a result, the prediction of potential therapeutic targets within this network requires the aid of systems biology-based and computational methods.

Uncovering major epigenetic features and the immune contexture of melanoma has catalyzed the development of anti-melanoma therapies within less than two decades. In 2004, no systemic therapies for melanoma had been shown to provide a survival benefit. Now, at least four regimens of targeted therapy and three for immunotherapy improve overall survival and disease-free survival, with each modality presenting distinct benefits and limitations. In particular, in 2011, vemurafenib became the first BRAF-targeted therapy 
approved by the Food and Drug Administration (FDA) for the treatment of melanoma [20]. Unfortunately, responses to BRAF inhibitor monotherapy, although impressive and rapid, were usually transient. In most cases, this was due to development of resistance via reactivation of the mitogen activated protein kinase (MAPK) pathway. Combined BRAF and MEK inhibition addresses this MAPK-mediated mechanism of resistance and constitutes the current standard-of-care for targeted melanoma therapy. Regimens of BRAF plus MEK inhibitors achieve longer-lasting disease control and are better tolerated than BRAF inhibitor monotherapy, but a major concern is that resistance, although delayed, is eventually developed [21,22]. Likewise, the treatment response of patients with mutant NRAS-positive metastatic melanoma to MEK inhibitors is transient and shortlived [23]. In the context of the paradigm-changing advances in cancer immunotherapy, several next-generation immune-based formulations, such as the checkpoint inhibitors ipilimumab, pembrolizumab, and nivolumab, have received FDA approval for the indication of metastatic melanoma and ensure durable responses. However, they are linked with immune-related toxicities and pose limitations for use in patients with either an overactive (autoimmune disease patients) or a suppressed (organ transplant recipients) immune system [24]. In summary, targeted therapy shows a rapid response time and generally less toxic off-target effects. However, resistance can be developed. While immunotherapy offers increased durability of benefit in all patients irrespective of tumor genotype, this is also frequently associated with immune-related side-effects, especially when combinations of immunotherapeutic drugs are used [21,22]. It is therefore essential to develop both, effective and safe strategies, that specifically interfere with the complex melanoma networks. This should enrich the antimelanoma drug arsenal with more personalized therapeutic options. The past decade's clinical experience taught us that, in general, combination therapies may be superior in terms of efficacy and/or safety than monotherapies. Combining anticancer drugs is currently seen as the approach most likely to overcome singleagent resistance, to produce sustained clinical remissions via multi-targeting effects on distinct mechanisms of action, and to reduce unwanted side-effects by usage of lower drug doses [25,26]. In fact, the need for combinatory therapies is an inevitable consequence of the evolving nature of tumors. Clonal evolution is particularly active when tumors are under selective pressures due to medical treatments, thereby promoting resistance to therapy. Resistant cell clones often preexist (although undetectable) at the start of treatment, supporting the idea that early administration of combinatorial treatments stands a higher chance of eradicating such clones when their number is very low, before acquired resistance is overtly diagnosed. Simultaneous targeting the driver oncogenic mutations along with the expected secondary resistance may provide a significant advantage in survival compared with administration at relapse. However, $a b$ initio combination therapies are challenging in the clinical oncology setting because of the narrow therapeutic window between tumor cells and host, which overall limits the number of agents that can be simultaneously tested [27]. With recent advances in high-throughput screening methods, a systematic evaluation of combinations among large collections of chemical compounds in vitro has become feasible. This typically requires large-scale experiments, in which the combinatorial responses are tested in various doses on cancer cell lines or patient-derived cells, resulting in dose-response matrices that capture the measured combination effects for every concentration pair in a particular sample [26]. For example, systematic screening of pairwise combinations of 104 FDA-approved oncology drugs has been performed in the NCI-60 panel of human tumor cell lines to produce a comprehensive platform for defining the pairs with enhanced therapeutic efficacy [25]. However, even with modern highthroughput instruments, experimental screening of drug combinations can become a 'Herculean task', as the number of conceivable drug combinations increases rapidly with the number of drugs under consideration. In addition, the inherent heterogeneity of cancer cells further challenges the experimental efforts, as the 
combinations need to be tested in various cell contexts and genomic backgrounds. Hence, computational methods are often recruited to guide the discovery of effective combinations that can be prioritized for further pre-clinical and clinical validation [26,28].

Herein, aided by in silico workflows, we sought to predict efficient and safe compounds that either alone or in combination prevent melanoma progression by specifically targeting components of the prometastatic E2F1-governed GRNs in melanoma. Using a comprehensive regulatory and functional map of E2F1 in tumor progression and metastasis [11] which contains different types of regulatory factors, including genes, proteins, microRNAs, or complexes, we identified a core regulatory network in melanoma [28,29]. The core regulatory network was subjected to logic-based modelling for detecting protein signatures which play an important role in interconnecting many of the responsive genes that are typically not identified through genebased differential expression analysis. Subsequent virtual screening, an increasingly technique that improves the speed and efficiency of the drug discovery process [30,31], was applied to find compound hits against protein signatures eliciting measurable biological responses and to estimate their safety profiles. Our approach predicted that MDM2 plus AKT1 inhibitors could be a promising combination that is worthwhile to be further investigated as a novel anti-metastatic regimen in high E2F1-expressing melanoma patients.

\section{Results}

2.1. Establishment of computational pipelines for prediction of drug-targetable components of the E2F1governed prometastatic GRN in melanoma and in silico screening of different inhibitors, alone or in combination

Previously, we have designed a comprehensive regulatory and functional map of E2F1 in tumor progression and metastasis [11] which contains different types of regulatory factors $(n=879)$ including genes, proteins, microRNAs, or complexes; and interactions $(\mathrm{m}=2278)$ based on information retrieved from published literature and databases. The map was modularized into three E2F1 regulatory compartments such as extra-/intracellular receptor signaling, post-translational modifications, regulators of E2F1 activity; and seven functional compartments including cell cycle, quiescence, DNA repair, metabolism, apoptosis, survival, and angiogenesis/invasion. Using a computational pipeline, we used the map to unravel a tumor type-specific regulatory core and to predict receptor protein signatures in bladder and breast cancer underlying E2F1mediated EMT transition. The E2F1 map and the previously used workflow is applied to identify a key functional module (core regulatory network) in melanoma. This core regulatory network is composed of regulatory motifs and critical molecular interactions that drive phenotype switching in melanoma. The core regulatory network for melanoma was subjected to our computational pipeline to detect protein signatures that play an important role in interconnecting many of the responsive genes that are typically not identified through gene-based differential expression analysis.

Our workflow includes (i) network-based analysis of topological parameters to characterize the pattern of factors in a networked system, (ii) mapping of the gene expression profiles from melanoma cell lines onto the E2F1 map, (iii) network reduction via a multi-objective function [32] to provide motif ranking by user-defined weights in an iterative manner, (iv) boolean modeling to analyze and predict the protein signatures linked to aggressiveness in melanoma, (v) virtual screening to find compound hits against protein signatures that elicit measurable biological responses, and (vi) to predict ADME behaviors, pharmacokinetic parameters, and druglikeness of compounds. The workflow in Figure 1 was used for prioritizing therapeutic targets and also to screen potential drug candidates that may be validated for the advanced melanoma tumors. 


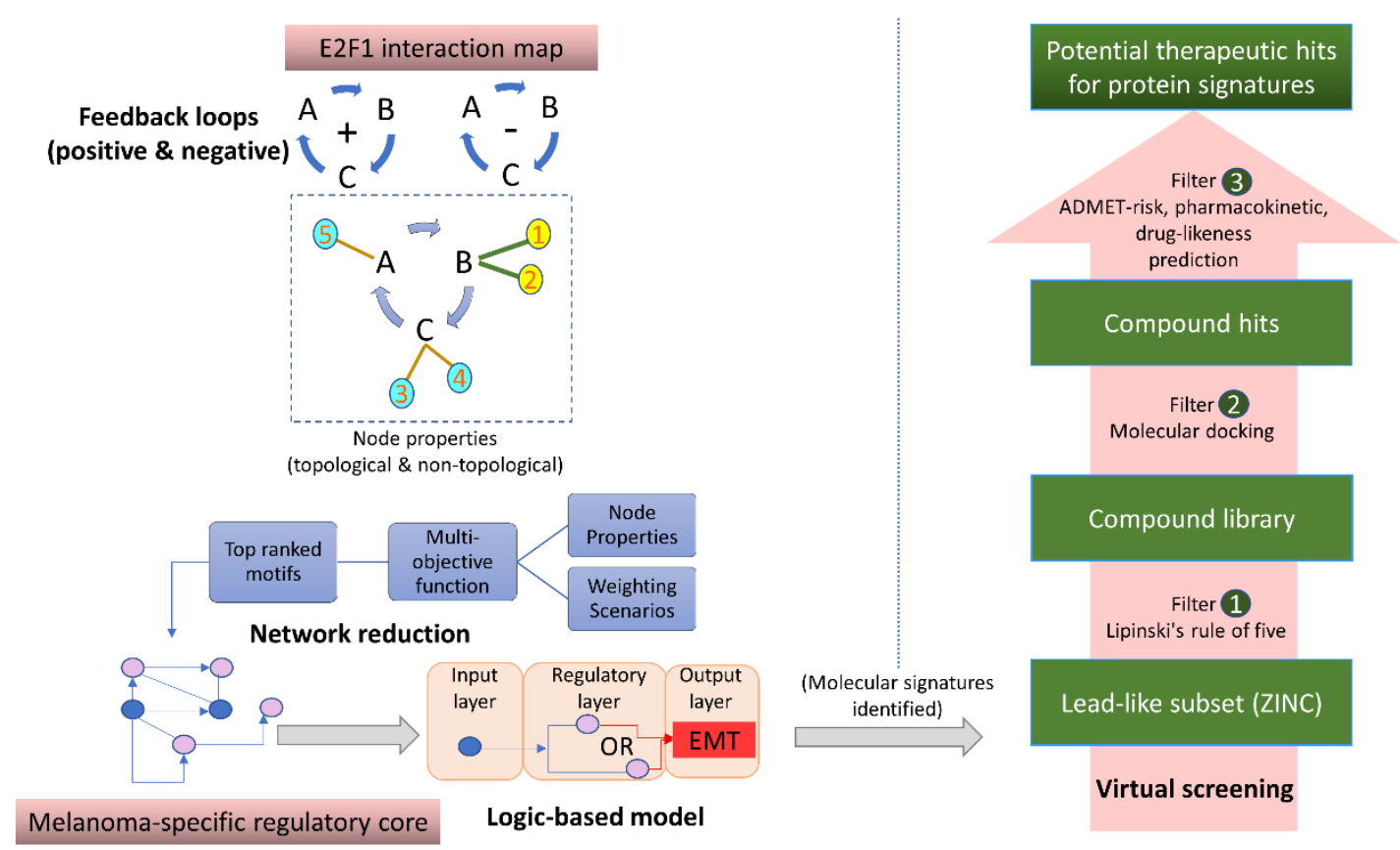

Figure 1: Workflow for the identification and prioritization of therapeutic targets regulating metastatic melanoma phenotypes and virtual screening of repurposed drugs. The overall workflow is divided into two parts. The left part focused on the identification of molecular signatures (mainly proteins) that drive melanoma metastasis. This was identified through the in silico perturbation experiments on melanoma-specific core regulatory network identified from the prioritized motifs from E2F1 interaction map. The right-hand side of the workflow highlights various filtering steps for the identification of small molecule inhibitors from large virtual libraries of lead-like substances from public databases, such as ZINC.

\subsection{Identification of the metastatic melanoma-specific core regulatory network}

We used our previously published network-based approach to construct a melanoma-specific regulatory core from the comprehensive map of E2F1 [11]. Here, we utilized the workflow and the E2F1 map to identify key network motifs and critical molecular interactions that drive a highly invasive melanoma cell phenotype. To do this, we have used the data extracted from the E2F1 map and identified important network motifs by calculation of topological and non-topological properties of each node (Supplementary file 1).

The motifs were prioritized based on a multi-objective optimization function where the function contains parameters accounting for each property. Weights are assigned to each property in terms of their importance in a user-defined manner and then, the motifs are ranked according to the value of the objective function. The top ten high-scored motifs were selected from each weighting scenario (Supplementary file 1). Finally, we merged all the top ranked motifs to obtain a melanoma-specific regulatory core. We expanded the regulatory core by adding receptor proteins which are the first neighbors of ranked motif nodes in the E2F1 map. We also added four well-known markers CDH1, VIM, ZEB1, and SNAI1 [10] in the core to measure the EMT response (Figure 2a). 


\subsection{Boolean modelling of the melanoma-specific core regulatory network}

We encoded the core-regulatory network into a Boolean model for stimulus-response and perturbation analyses. Stimulus-response analysis was used to identify the effect of up/down expressed receptors on the EMT phenotype, and perturbation analysis predicted potential drug target that can bring the phenotype to lower possible level. In Boolean model, state a node is represented into one of two possible states i.e., 0 (OFF, inactive) or $1(\mathrm{ON}$, active) [33]. The regulatory relationships between upstream nodes (i.e., sources) to downstream nodes (i.e., targets) are encoded into Boolean functions using logical gates 'NOT', 'OR' and 'AND'. Further, we calibrated the Boolean functions with fold-change (FC) expression data [11] of publicly available dataset GSE46517 [34] from Gene Expression Omnibus (GEO).

To evaluate the input-output behavior, we divided the model into three layers: 1) Input layer, containing receptor molecules, 2) regulatory layer, comprising nodes constituting a core-regulatory network, and 3) Output layer, including EMT phenotype (Figure 2b). Input layer was initialized with FC expression profile i.e., a node with negative FC was represented by a state 0 and a node with positive FC was represented by a state 1 .

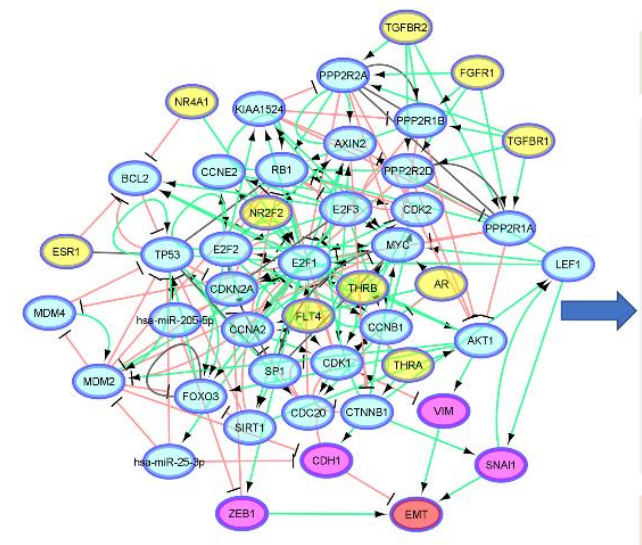

(a)

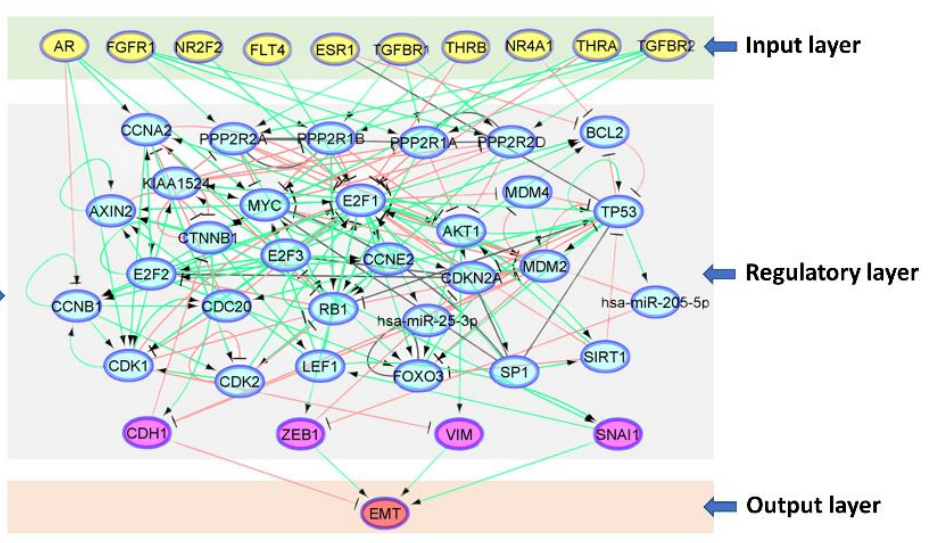

(b)

Figure 2: Melanoma-specific core regulatory network. (a) The core regulatory network was derived by merging the topranked motifs from the E2F1 map. The constructed core consists of 183 direct interactions (edges) involving 34 core proteins and miRNAs, 10 receptor proteins, and 4 EMT marker proteins. Regulatory directions were retrieved from the E2F1 map as activation (+1), inhibition (-1), and unidentified (0). (b) Boolean model of the regulatory network. Model is divided into three layers: input layer containing receptor molecules (green background), regulatory layer comprising the regulatory network nodes (gray background) and output layer containing the EMT phenotype (red background). Green color edges represent activation, red color edges represent inhibition and gray edges represent neutral regulatory relationships among the nodes. Description of gene symbols appearing in the network: AKT1: AKT serine/threonine kinase 1, AR: androgen receptor, AXIN2: axin 2, BCL2: BCL2 apoptosis regulator, CCNA2: cyclin A2, CCNB1: cyclin B1, CCNE2: cyclin E2, CDC20: cell division cycle 20, CDH1: cadherin 1, CDK1: cyclin dependent kinase 1, CDK2: cyclin dependent kinase 2, CDKN2A: cyclin dependent kinase inhibitor 2A, CTNNB1: catenin beta 1, E2F1: E2F transcription factor 1, E2F2: E2F transcription factor 2, E2F3: E2F transcription factor 3, ESR1: estrogen receptor 1, FGFR1: fibroblast growth factor receptor 
1, FLT4: fms related receptor tyrosine kinase 4, FOXO3: forkhead box O3, KIAA1524: cancerous Inhibitor Of Protein Phosphatase 2A, LEF1: lymphoid enhancer binding factor 1, MDM2: MDM2 proto-oncogene, MDM4: MDM4 regulator of p53, MYC: MYC proto-oncogene, bHLH transcription factor, NR2F2: nuclear receptor subfamily 2 group F member 2, NR4A1: nuclear receptor subfamily 4 group A member 1, PPP2R1A: protein phosphatase 2 scaffold subunit A alpha, PPP2R1B: protein phosphatase 2 scaffold subunit A beta, PPP2R2A: protein phosphatase 2 regulatory subunit B alpha, PPP2R2D: protein phosphatase 2 regulatory subunit B delta, RB1: RB transcriptional corepressor 1, SIRT1: sirtuin 1, SNAI1: snail family transcriptional repressor 1, SP1: Sp1 transcription factor, TGFBR1: transforming growth factor beta receptor 1, TGFBR2: transforming growth factor beta receptor 2, THRA: thyroid hormone receptor alpha, THRB: thyroid hormone receptor beta, TP53: tumor protein p53, VIM: vimentin, ZEB1: zinc finger E-box binding homeobox 1

\subsection{In silico perturbation simulations using Boolean modeling}

Model was simulated with initial values derived from the expression profile of input nodes and confirmed that the logical state of nodes in a regulatory layer represents the data (for details see Supplementary file 2). The EMT phenotype was regulated by nodes ZEB1, CDH1, VIM and SNAI1 [10], and represented by 5 ordinal level ranking from 0 (minimum) to 4 (maximum):

$$
\mathrm{EMT}=\mathrm{ZEB} 1+\mathrm{NOT}(\mathrm{CDH} 1)+\mathrm{VIM}+\mathrm{SNAI} 1
$$

For the initial condition, model simulations result in EMT of level 3, where ZEB1 and SNAI1 are active and CDH1 is inactive (see Table 1a). Further, we performed perturbation analysis of all nodes (except ZEB1, CDH1, VIM and SNAI1) in the regulatory layer of the model to bring EMT from level 3 to minimum level. We identified that for a single perturbation (in this case inhibition) of MDM2 or MIR25, EMT can be reduced to level 1 (see Table 1b). CDH1 is activated upon inhibition of MDM2 which inhibit EMT as well as inhibit CTNNB1 which subsequently inhibit SNAI1 [35,36] to further reduce EMT. Similar effect was observed upon inhibition of MIR25 [37,38]. On the other hand, single perturbation (in this case activation) of AKT1 can increase the EMT to the highest level 4.

Table 1: Stimulus-response and perturbation simulation results. (a) Model simulation results of initial condition which results in higher EMT level. (b) Single perturbation of MDM2 or MIR25 can bring EMT from level 3 to 1 .

\begin{tabular}{|c|c|c|c|c|c|c|c|c|c|c|c|c|c|c|c|c|c|}
\hline & \multicolumn{17}{|c|}{ (a) Stimulus-response analysis for initial condition } \\
\hline AR & ESR 1 & FGFR1 & FLT4 & NR2F2 & NR4A1 & TGFBR1 & TGFBR2 & THRA & THRB & MDM2 & MIR25 & AKT! & ZEB1 & $\mathrm{CDH} 1$ & VIM & SNAI1 & EMT \\
\hline $\mathrm{NaN}$ & 1 & 1 & $\mathrm{NaN}$ & 1 & 1 & 1 & $\mathrm{NaN}$ & 0 & $\mathrm{NaN}$ & 1 & 1 & 0 & 1 & 0 & 0 & 1 & 3 \\
\hline & \multicolumn{17}{|c|}{ (b) Single perturbations analysis (inhibition of MDM2, MIR25 and activation of AKT1) for EMT level of 3} \\
\hline AR & ESR1 & FGFR1 & FLT4 & $\mathrm{NR} 2 \mathrm{~F} 2$ & NR4A1 & TGFBR1 & TGFBR2 & THRA & THRB & MDM2 & MIR25 & AKT1 & ZEB1 & $\mathrm{CDH} 1$ & VIM & SNAI1 & EMT \\
\hline $\mathrm{NaN}$ & 1 & 1 & $\mathrm{NaN}$ & 1 & 1 & 1 & $\mathrm{NaN}$ & 0 & $\mathrm{NaN}$ & 0 & 1 & 0 & 1 & 1 & 0 & 0 & 1 \\
\hline $\mathrm{NaN}$ & 1 & 1 & $\mathrm{NaN}$ & 1 & 1 & 1 & $\mathrm{NaN}$ & 0 & $\mathrm{NaN}$ & 1 & 0 & 0 & 1 & 1 & 0 & 0 & 1 \\
\hline $\mathrm{NaN}$ & 1 & 1 & $\mathrm{NaN}$ & 1 & 1 & 1 & $\mathrm{NaN}$ & 0 & $\mathrm{NaN}$ & 1 & 1 & 1 & 1 & 0 & 1 & 1 & 4 \\
\hline
\end{tabular}

\subsection{Assessment of protein signatures identified through Boolean modelling}

Our Boolean model simulations suggested two key proteins AKT1 and MDM2 that upon inhibition can bring the EMT from level 3 to 1 . Interestingly, AKT1 directly activates the VIM, a key marker for EMT. AKT1 also activate MDM2 which interact with p53 to regulate immune axis in the metastatic melanoma. MDM2 also 
indirectly activate the EMT by downregulating another hallmark protein CDH1. We investigated the expression profiles of AKT1 and MDM2 and their impact on melanoma patient survival using Kaplan-Meier curve (Figure 3) using TCGA melanoma SKCM dataset (https://portal.gdc.cancer.gov/projects/TCGA-SKCM). We found that higher expression of both AKT1 and MDM2 resulted in poor patient survival. These observations also confirm that the Boolean model simulation were successful in predicting potential proteins that may be targeted for the treatment of metastatic melanoma.

(a) AKT1

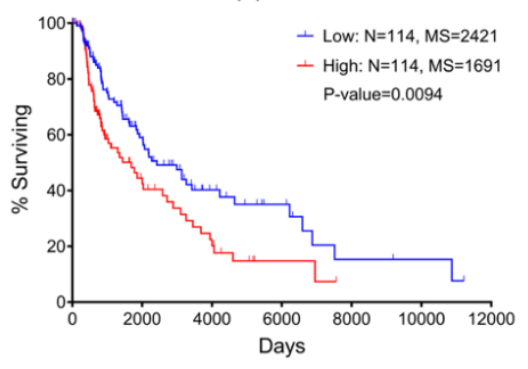

(b) MDM2

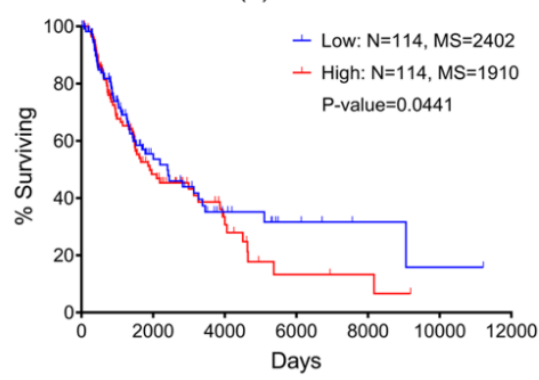

(c) AKT1-MDM2

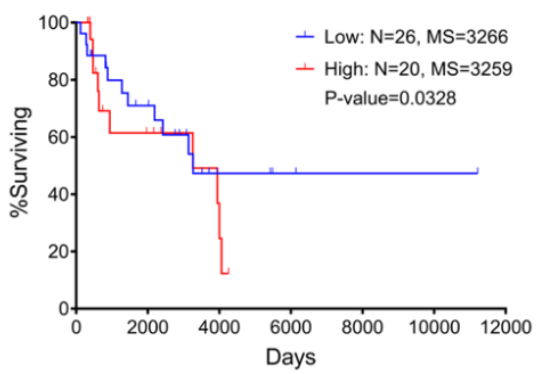

Figure 3: Kaplan-Meier plots suggest that patient with high expression of (a) AKT1; (b) MDM2; and (c) high expression of AKT1 and MDM2 together have worst clinical outcomes.

\subsection{Screening of small molecule inhibitors to block protein signatures}

To identify compound drugs that are most likely to bind to AKT1 and MDM2 protein signatures, molecular docking was performed with the filtered library compounds (Supplementary file 3 ). The information about the active sites of proteins is retrieved from the literature and PDB database. More specifically, for AKT1 we performed screening against the kinase domain (150-408) which is previously selected to identify ATPcompetitive inhibitors [39,40] (Figure 4a). For MDM2, many recent studies indicate that its overexpression and subsequent deactivation of p53 result in failure of apoptosis and cancer cell survival [41-43]. We investigated the p53-Mdm2 interaction surface which is $~ 700 \AA^{2}$. This druggable pocket of MDM2 where p53 binds provides a great opportunity for compound inhibitors to disrupt p53-MDM2 interaction [44] (Figure 4b). 


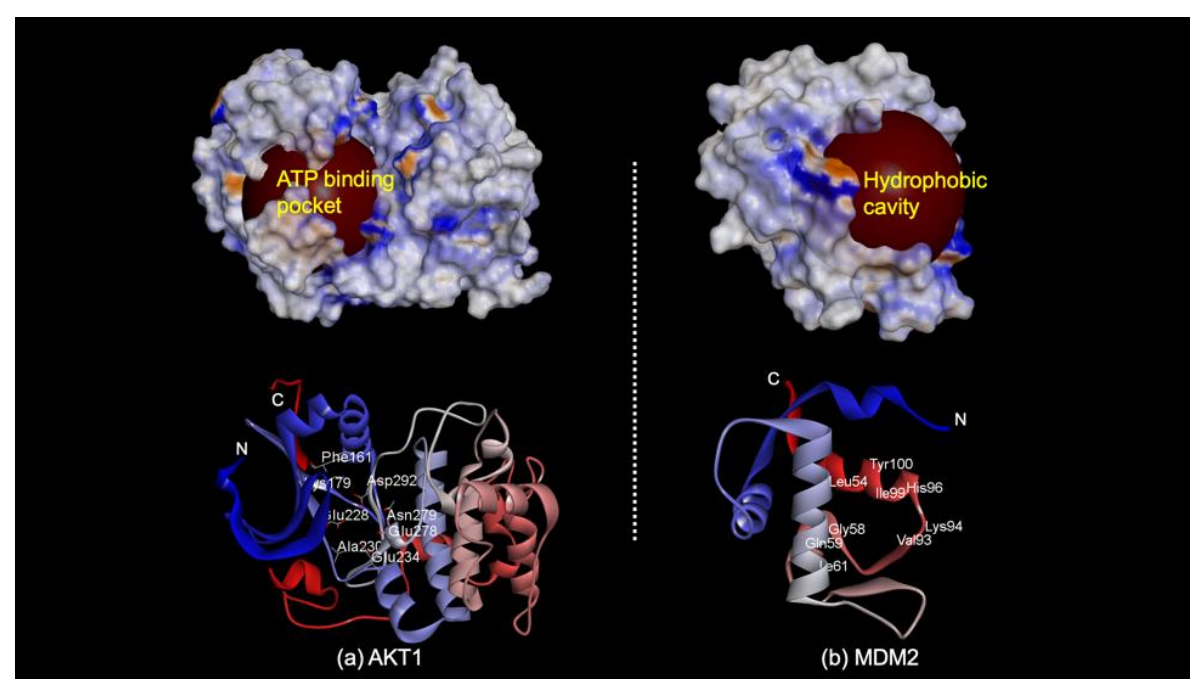

Figure 4: Functional binding sites of AKT1 and MDM2 used in the screening of drug library. On the top the surface model of AKT1 and MDM2 are shown along with the binding pocket. In case of MDM2 (PDB: 3JZK_chain A), the binding site is identified as main hydrophobic cavity that interacts with p53 and in case of AKT1 (PDB: 3OCB_chainA), the kinase domain showing the ATP binding pocket, in red spheres respectively. In the bottom, key amino acid residues participating in the binding pocket formation are shown.

The Achieved docked poses were ranked by their docking binding energies and were further analyzed to obtain the best conformation and orientation of the ligand in the active site.

In case of AKT1, docking analyses revealed that the compounds are packed against the residues LEU156, GLY157, PHE161, VAL164, ALA177, LYS179, GLU191, HIS194, GLU198, TYR229, ALA230, GLU234, ASP274, ASN279, MET281, ASP292, GLY294, LEU295, TYR437, PHE438, ASP439, and PHE442 and was stabilized by the hydrogen bonds, electrostatic, hydrophobic, and van der walls interactions.

Three hits namely, ZINC000043178353 [45], ZINC000040429080 [46], and ZINC000043202934 [47] are reported as selective AKT1 inhibitors with IC50 values of 0.5, 0.03, and 1.0 nM respectively, and displayed potency against AKT1, AKT2, and AKT3 within cells [40,47]. However, we found two novel hits (ZINC000001491367: binding energy -12.6 kcal/mol; ZINC000003939645: binding energy $-12.5 \mathrm{kcal} / \mathrm{mol}$ ) that were not investigated as AKT1 inhibitors before. Their binding energies towards the ATP binding pocket of AKT1 is comparable to ATP competitive inhibitors [40]. These two novel hits are reportedly inhibitors of CDKs and their cyclin partners, particularly CDK7/cyclin $\mathrm{H}$ and CDK2/cyclin E which are often deregulated in cancer. Both hits showed a considerable activity when compared with Seliciclib, a drug in phase II clinical trial for the treatment of cancer [48]. Interestingly, the number of interactions that strongly bind these two compounds into the cavity was also reasonably high as compared to other hits (Figure 5).

To determine the difference between the binding mode of novel hits to AKT1 from that of previously known inhibitors, a comparative analysis was performed. It was found that the common interacting residues in all inhibitors were GLU234 and ASP292. The amino acid residue GLU234 of the protein backbone is necessary for the AKT1 biological activity and this interaction was found with most of the previously known ATPcompetitive kinase inhibitors [49,50]. The second set of electrostatic interactions and hydrogen bonds to ASP292 in AKT1 is critical because this position is typically occupied by a divalent cation (Mg2+) bound to ATP [50]. Other common amino acid residues for all the five hits were PHE161 and LYS179; which indicates that the top screening hits ZINC000001491367 and ZINC000003939645 are reliable and promising for further evaluation. 


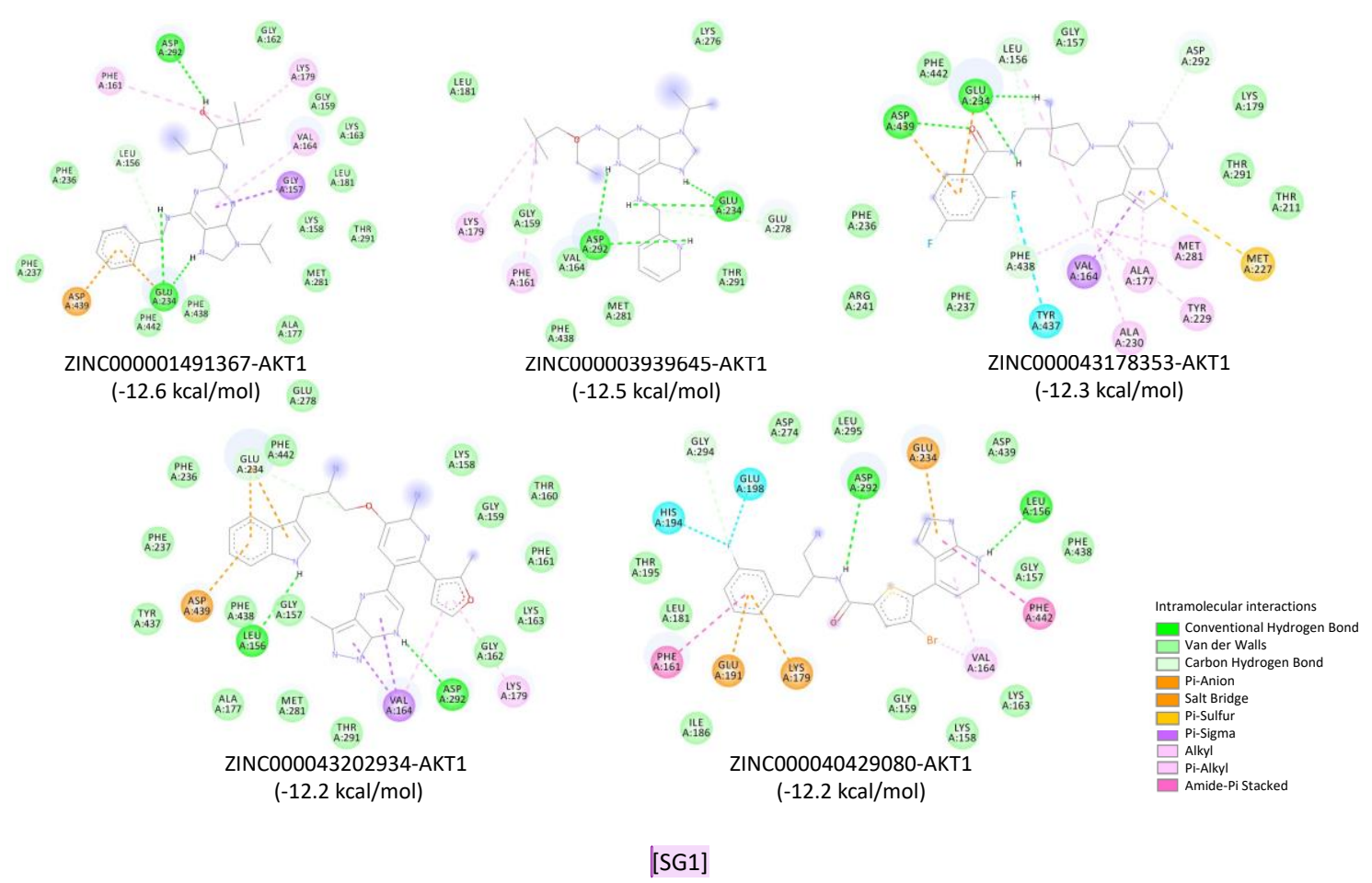

Figure 5: 2D interaction diagrams for docking poses of AKT1 with top five screened hits: ZINC000001491367, ZINC000003939645, ZINC000043178353, ZINC000043202934, and ZINC000040429080

In case of MDM2, the molecular docking was performed into lining residues of this pocket containing amino acids (LEU54, LEU57, ILE61, MET62, TYR67, GLN72, VAL75, PHE86, PHE91, VAL93, HIS96, ILE99, TYE100, and ILE101). These residues form a hydrophobic cavity on the MDM2 protein structure and are potentially occupied by known inhibitors [51,52]. The docked compound hits bind to MDM2 by interacting with key residues and particularly, hydrophobic interaction with residues VAL93 and LEU54 can be seen in all five hits (Figure 6).

The first compound hit ZINC000000537755 (Fluspirilene), exhibits a convincing binding mode into the MDM2 pocket. Fluspirilene is reported to show anti-proliferative activity at $10 \mu \mathrm{m}$ in the NCI60 tumor cell line [53]. The second hit ZINC000169352550 is a compound containing morpholinone which is highly potent and selective MDM2 inhibitors [44]. The third hit, ZINC000095605306 is a close analog of trans-morpholinone and binds to the protein in the same mode as inhibitors. The fourth hit, ZINC000084689539 (AM-8553) interact with the three key p53 binding residues (PHE19, TRP23, and LEU26) in the pocket. The compound is effective in the inhibition of tumor growth in the SJSA-1 mouse xenograft model [44,54]. The two hits, ZINC000169352550 and ZINC000096286451 showed p53 ubiquitination in vitro with IC50 values of $0.84 \mu \mathrm{m}$ and $1.5 \mu \mathrm{m}$ [55], respectively. All compound hits showed good complementarity within the binding pocket. More specifically, ZINC000095605306 and ZINC000084689539 forms hydrogen bond interactions with hydrophobic residues PHE55 and GLY58; and LEU54 respectively, suggesting a stable binding of compounds to the p53-binding pocket on MDM2. 


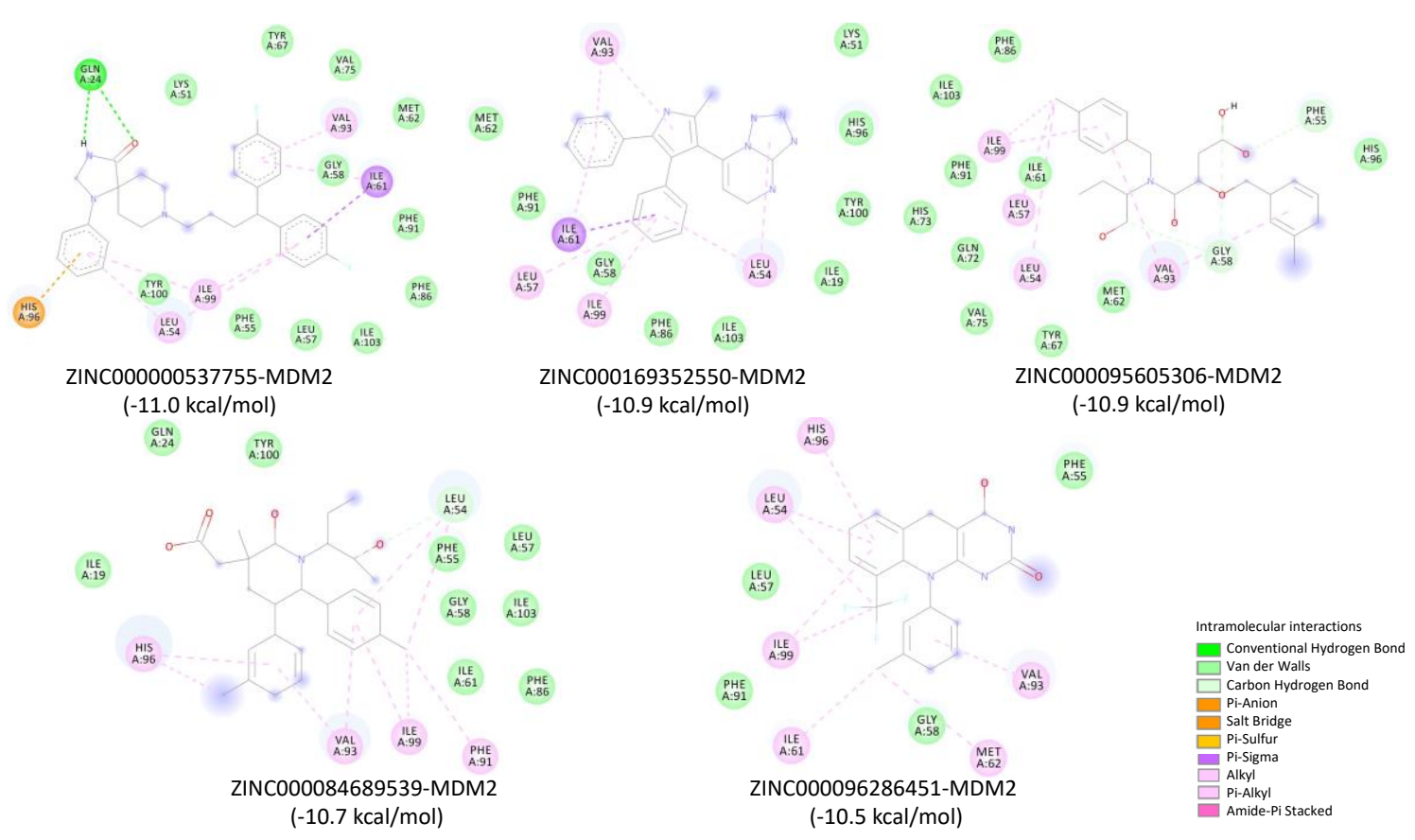

Figure 6: 2D interaction diagrams for docking poses of MDM2 with top five screened hits: ZINC000000537755, ZINC000169352550, ZINC000095605306, ZINC000084689539, and ZINC000096286451.

\subsection{ADME/pharmacokinetic predictions and drug-likeness}

Bioavailability:

The bioavailability radar plots (Figure 7) show a rapid appraisal of drug-likeness based on the physicochemical properties of the lead molecules. In the graphical output, the radar area (pink color) is in the optimal range for compound hits ZINC000001491367, ZINC000003939645, ZINC000043178353, ZINC000000537755, ZINC000095605306, and ZINC000084689539 giving information that the hits are falling entirely within the physicochemical range on each axis and could be considered drug-like. However, the compounds hits ZINC000040429080, ZINC000043202934, ZINC000000537755, and ZINC000096286451 are predicted to be not orally bioavailable because they are too polar and fraction Csp3 (in-saturation) is too high. 


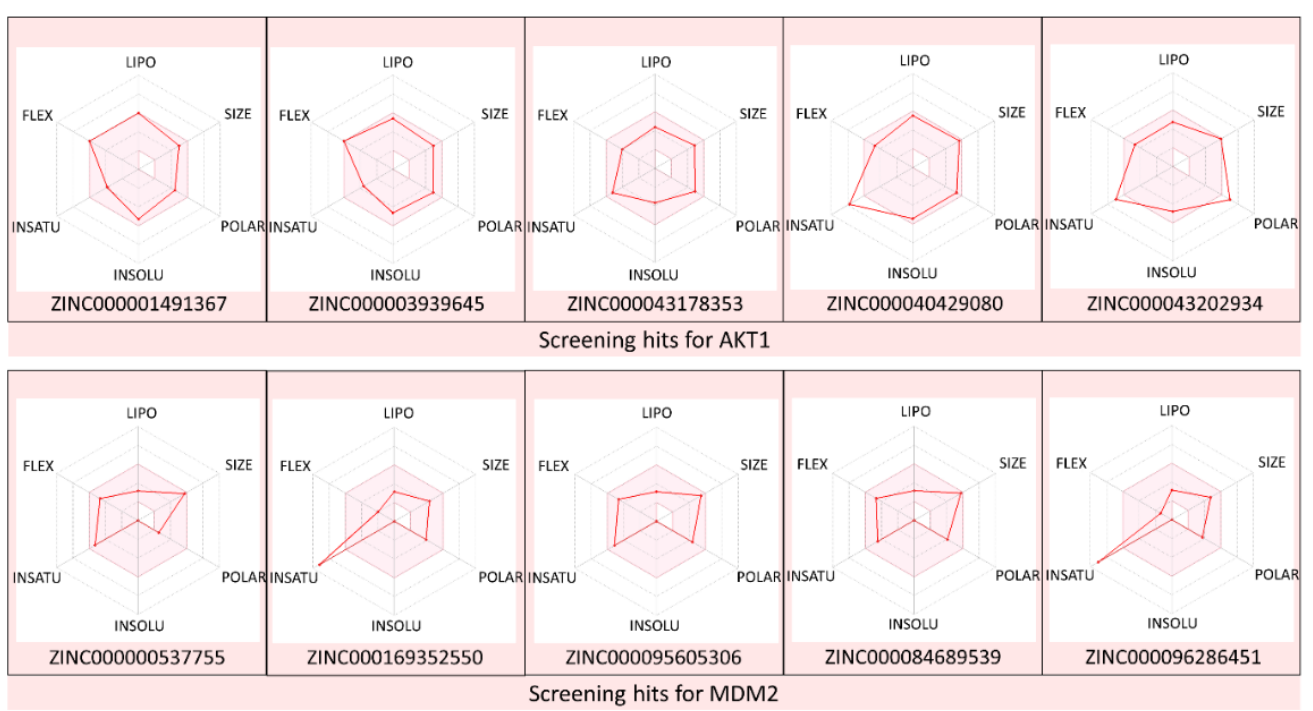

Figure 7: Radar plots of screened hits for oral bioavailability based on physicochemical properties LIPO (lipophilicity), SIZE (molecular weight), POLAR (topological polar surface area), INSOLU (insolubility), INSATU (in-saturation), and FLEX (flexibility). The pink-colored area represents the ideal range for each property i.e. XLOGP3 $(-0.7$ and +5.0$)$, MW (150 and 500 g/mol), TPSA (20 and $130 \AA 2$ A), Log S (<6), Fraction Csp3 (<1), and Rotatable bonds (<9), respectively.

Bioaccumulation:

As shown in Table 2, the compound hits ZINC000001491367, ZINC000003939645, ZINC000095605306, ZINC000084689539, and ZINC000096286451 did not exhibit in silico inhibition of CYP2C9 and CYP2D6, members of the drug-metabolizing cytochrome P450 family of enzymes [49,56]. Though, other hits are interacting with CYP isoenzymes which could lead to bioaccumulation of compounds and toxicity.

Aqueous solubility and gastrointestinal absorption:

The aqueous solubility for all hits is estimated to be moderate except ZINC000000537755 which is predicted to be poorly soluble. Prediction of passive gastrointestinal absorption (GIA) was high for all the hits except ZINC000043202934 and it is based on the Intestinal Estimated permeation model [57]. It is observed that hits ZINC000001491367 and ZINC000003939645 are non-substrate to P-glycoprotein (multidrug resistance protein in the cell membrane) [58], suggesting that they are likely to have high intestinal absorption and bioavailability. Compliance with Lipinski’s, Ghose, Veber, Egan, and Muegge rules associated with drug-likeness:

Moreover, compound hits ZINC000001491367, ZINC000003939645, ZINC000043178353, ZINC000040429080, ZINC000095605306, ZINC000084689539, and ZINC000096286451 are not violating the Lipinski’s, Ghose, Veber, Egan, and Muegge rules. All compound hits except ZINC000096286451 have passed the PAINS and Brenk filters for not containing any problematic fragments. In addition, toxicity profiles [59] such as carcinogenicity, immunotoxicity, mutagenicity, and cytotoxicity profiles of ZINC000001491367, ZINC000003939645, ZINC000095605306, ZINC000084689539, and ZINC000096286451 are predicted to be inactive. Overall, the results of the ADME and drug-likeness reveals that ZINC000001491367, ZINC000003939645 (AKT1 hits), and ZINC000095605306 and ZINC000084689539 (MDM2 hits) show good pharmacokinetic properties and are predicted to be orally bioavailable, non-toxic, and good absorption. 
2 Table 2: Prediction of ADMET, pharmacokinetic, drug-likeness properties and medicinal friendliness of the screened hits for AKT1 and MDM2.

\begin{tabular}{|c|c|c|c|c|c|c|c|c|c|c|c|c|c|c|c|c|c|}
\hline \multirow{2}{*}{$\begin{array}{l}\text { Protein } \\
\text { Signatures }\end{array}$} & \multirow[t]{2}{*}{ ZINC ID } & \multirow{2}{*}{$\begin{array}{c}\text { Water } \\
\text { solubility }\end{array}$} & \multirow{2}{*}{$\begin{array}{c}\text { Medicinal } \\
\text { Chemistry } \\
\text { PAINS \& } \\
\text { BRENK } \\
\text { alerts }\end{array}$} & \multicolumn{5}{|c|}{ Drug-likeness (\#violations) } & \multicolumn{5}{|c|}{ Pharmacokinetics } & \multicolumn{4}{|c|}{ Toxicity profile } \\
\hline & & & & Lipinski & Ghose & Veber & Egan & Muegge & $\begin{array}{c}\text { Gastro- } \\
\text { intestinal } \\
(\mathbf{G I}) \\
\text { absorption }\end{array}$ & $\begin{array}{c}\text { Blood- } \\
\text { brain } \\
\text { barrier } \\
\text { (BBB) } \\
\text { permeant }\end{array}$ & $\begin{array}{c}\text { P- } \\
\text { glycoprotein } \\
\text { (Pgp) } \\
\text { substrate }\end{array}$ & $\begin{array}{l}\text { CYP2C9 } \\
\text { inhibitor }\end{array}$ & $\begin{array}{l}\text { CYP2D6 } \\
\text { inhibitor }\end{array}$ & 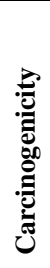 & 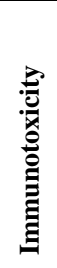 & 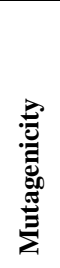 & 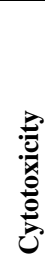 \\
\hline \multirow[t]{5}{*}{ AKT1 } & ZINC000001491367 & Moderate & 0 & 0 & 0 & 0 & 0 & 0 & High & No & No & No & No & $x$ & $x$ & $x$ & $x$ \\
\hline & ZINC000003939645 & Moderate & 0 & 0 & 0 & 0 & 0 & 0 & High & No & No & No & No & $x$ & $x$ & $x$ & $x$ \\
\hline & ZINC000043178353 & Moderate & 0 & 0 & 0 & 0 & 0 & 0 & High & No & Yes & No & Yes & $x$ & $\checkmark$ & $x$ & $x$ \\
\hline & ZINC000040429080 & Moderate & 0 & 0 & 0 & 0 & 0 & 0 & High & No & Yes & Yes & Yes & $x$ & $\checkmark$ & $x$ & $x$ \\
\hline & ZINC000043202934 & Moderate & 0 & 0 & 2 & 1 & 1 & 1 & Low & No & Yes & Yes & Yes & $\checkmark$ & $\checkmark$ & $\checkmark$ & $x$ \\
\hline \multirow[t]{5}{*}{ MDM2 } & ZINC000000537755 & Poor & 0 & 1 & 1 & 0 & 0 & 1 & High & Yes & Yes & Yes & Yes & $x$ & $\checkmark$ & $x$ & $x$ \\
\hline & ZINC000169352550 & Moderate & 0 & 0 & 0 & 0 & 0 & 0 & High & Yes & Yes & No & No & $x$ & $x$ & $x$ & $x$ \\
\hline & ZINC000095605306 & Moderate & 0 & 0 & 0 & 0 & 0 & 0 & High & No & Yes & No & No & $x$ & $x$ & $x$ & $x$ \\
\hline & ZINC000084689539 & Moderate & 0 & 0 & 0 & 0 & 0 & 0 & High & No & Yes & No & No & $x$ & $x$ & $x$ & $x$ \\
\hline & ZINC000096286451 & Moderate & 1 & 0 & 0 & 0 & 0 & 0 & High & No & No & No & No & $x$ & $x$ & $x$ & $x$ \\
\hline
\end{tabular}

ZINC000001491367: BDBM50358241; (3R,4S)-4-[[6-(benzylamino)-9-isopropyl-purin-2-yl]amino]-2,2-dimethyl-hexan-3-ol

ZINC000003939645: BDBM50358233; 4-[9-isopropyl-6-(2-pyridylmethylamino)purin-2-yl]amino-2,2-dimethyl-hexan-3-ol

ZINC000043178353: PF-AKT400; AKT-0286; CS-5109; BDBM50322393; N-\{[(3s)-3-amino-1-(5-ethyl-7h-pyrrolo[2,3-D]pyrimidin-4-Yl)pyrrolidin-3-Yl]methyl\}-2,4-difluorobenzamide

ZINC000040429080: BDBM50278836; N-((S)-1-amino-3-(3-fluorophenyl)propan-2-yl)-4-bromo-5-(1H-pyrrolo[2,3-b]pyridin-4-yl)thiophene-2-carboxamide

ZINC000043202934: BDBM50306157; 3-((S)-2-amino-3-(1H-indol-3-yl)propoxy)-5-(3-methyl-1H-pyrazolo[4,3-b]pyrazin-5-yl)-6-(2-methylfuran-3-yl)pyridin-2-amine

ZINC000000537755: Fluspirilene; Redeptin; BDBM26948; 8-[4,4-bis(4-fluorophenyl)butyl]-1-phenyl-1,3,8-triazaspiro[4.5]decan-4-one

ZINC000169352550: BDBM50020715; 7-(2-methyl-4,5-diphenyl-1H-pyrrol-3-yl)tetrazolo[1,5-a]pyrimidine

ZINC000095605306: BDBM50432652; 2-[(2S,5R,6S)-6-(3-chlorophenyl)-5-(4-chlorophenyl)-4-[(1S)-1-(hydroxymethyl)propyl]-3-oxo-morpholin

ZINC000084689539: AM-8553; BDBM50388626; \{(3r,5r,6s)-5-(3-chlorophenyl)-6-(4-chlorophenyl)-1-[(2s,3s)-2-hydroxypentan-3-Y1]-3-methyl-2-oxopiperidin-3-Y1 $\}$ acetic acid

ZINC000096286451: BDBM50442784; 10-(3-chlorophenyl)-9-(trifluoromethyl)pyrimido[4,5-b]quinoline-2,4-dione 


\section{Discussion}

A common approach to anticancer drug development has been based on a workflow, whereby molecules that are designed from scratch, to specifically interfere with a certain pathway, are anticipated to target and eradicate tumors in a highly selective manner, analogous to the "lock-and-key" specificity, hence maximizing efficacy and minimizing side effects [60]. Despite their promising results in the preclinical setting, the majority of innovative drugs are proven insufficient or suboptimal when administered in clinical patients, thereby leading to unacceptably low success rates of clinical trials $[61,62]$. The high failure rate of this approach is the consequence of several unpredictable parameters, mainly: (a) the individual genetic background of cancer patients, which limits the therapeutic benefits only to specific patient subpopulations and necessitates treatment personalization [22]; (b) the fact that cancer-related genes are highly interconnected and regulate each other through complex loops from different pathways [63-65]; (c) the inherent ability of tumors to adapt and evolve, which catalyzes acquisition of resistance to therapies, especially monotherapies [27]. To address these challenges, computational methodologies including, but not limited to, algorithms and machine learning tools, are now being increasingly recruited in many drug discovery programs. For example, computational approaches that 'dock' small molecules into the structures of macromolecular targets and 'score' their potential complementarity to binding sites are widely used in hit identification and lead optimization and are currently reforming the pharmacopeia landscape [66]. This approach allows for fast and comprehensive screening of the efficacy and safety profiles of a high number of leads, in the context of a particular cancer type. Prioritization of the top-resulting leads or combinations thereof could subsequently facilitate faster introduction to clinical trials and significantly reduce the costs for drug development.

Having in mind that metastasis is linked with activation of E2F1-governed GRNs, we applied a transcriptomics-aided bioinformatics workflow, followed by virtual drug screening to comprehensively characterize novel therapeutic targets in melanoma and predict their corresponding drug inhibitors. Due to the documented ability of targeted drugs to show superior safety and efficacy in combination schemes [22], we were particularly interested on drugs that can perturb these prometastatic GRNs when used simultaneously. Using a well-established E2F1 map [11], we derived a set of three-node FBLs $(n=44)$ and used a ranking scheme that applies a weighted multi-objective function integrating topological and non-topological properties of each node. Topological properties such as node degree (number of edges connected to the node) are known for their importance in network organization and playing as central hubs in orchestrating molecular connections [67]. It is reported that cancer-associated proteins have large betweenness centrality as they control the communication between different components of a network [68]. Among non-topological properties, we have calculated the involvement of the motif constituents in the disease pathway, the gene prioritization score, and average $\log 2$ fold change for each motif based on the change in expression values of each node from noninvasive to invasive phenotypes derived from in vitro experiments. Since the network was originally constructed around E2F1, the topological properties for some nodes are expected to be higher than other nodes. Therefore, to give equal importance to all nodes, we used different weighting scenarios in the multi-objective optimization function to avoid biases and ranked motifs accordingly. The top ranked motifs are merged to understand their combined effect on the regulation of EMT in melanoma. We further expanded the regulatory core network by 
adding receptor proteins the first neighbours of the ranked motif nodes and four marker proteins and their direct connections from the E2F1 map. Receptor proteins work as determinative factors and markers proteins are required to measure the EMT response. We developed a three-layered logic-based model of the regulatory core consisting of an input layer, a regulatory layer, and an output layer. We analyzed the regulatory core by using boolean logic for the input and regulatory layers, and multi-valued logic for the output layer which allows us to assess the combined effect of various network components on the EMT phenotype. Our model simulations identified two protein signatures AKT1 and MDM2 as potential drivers of EMT in melanoma. Further virtual drug screening with particular emphasis on the prediction of compounds with minimal toxicities revealed that AKT1 and MDM2 inhibitors, either alone or in combination with each other, can efficiently and safely suppress E2F1-driven invasion in melanoma.

The PI3K-AKT signaling pathway is predominantly activated in melanomas, upon mutations of crucial melanoma drivers, in parallel with the MAPK (Ras-Raf-MEK-ERK) pathway. The BRAF/MEK inhibitors, which constitute the standard-of-care in the targeted therapeutics of melanoma, suppress the MAPK pathway. However, an active PI3K-AKT pathway offers alternative avenues for evasion of MAPK-targeting regimens and disease progression. Indeed, members of the phosphatidylinositol 3-kinase (PI3K)/AKT pathway are implicated in melanoma progression, metastasis, and acquired resistance to MAPK-targeting therapies. AKT1 activation frequently occurs through silencing of PTEN, a tumor-suppressor gene encoding a phosphatase which acts on lipid and protein substrates. The major PTEN substrate is phosphatidylinositol-3,4,5-triphosphate (PIP3), which recruits AKT to the membrane and activates it by phosphorylation. By specifically dephosphorylating PIP3, PTEN suppresses the downstream signaling of AKT and, vice-versa its loss results in increased PIP3 levels and subsequent AKT1 activation. Combination of PTEN silencing with BRAFV600E expression in vivo leads to melanoma formation, while further ectopic expression of activated AKT1 in this genetic context co-operates with PTEN silencing to accelerate metastasis to lungs and brain [69]. Progressing melanomas, in their vast majority, develop resistance to therapy due to reactivation of MAPK signaling, commonly via alterations in BRAF, NRAS and MEK1/2. However, a small proportion of resistant melanomas rely on the activation of the compensatory PI3K/AKT signaling cascade. PI3K/AKT-activating mutations in melanoma do not override proliferative arrest induced by BRAF/MEK inhibition, but rather enable the survival of a small dormant subpopulation of MAPK-inhibited melanoma cells. The persistent exposure of this cell subpopulation to the constant selective pressure of BRAF/MEK inhibition eventually promotes reorganization of signaling circuits and accelerates the evolution of tumor subclones that are highly resistant to targeted therapies [70]. Upregulation of the PI3K-AKT pathway is a critical event during the early and late evolution of resistance to MAPK pathway inhibition [71]. It has been proposed that AKTi combined with BRAFi-based therapy may benefit patients with tumors harboring BRAF mutations along with PTEN deletions or AKT mutations [72]. In agreement with these studies, our analysis highlighted inhibition of AKT1 as an attractive strategy for preventing EMT-driven metastatic progression of melanomas with a high-E2F1 content.

In addition to the PI3K/AKT pathway, the manipulation of the p53-controlled pathways is emerging as an alternative therapeutic option with a potential to overcome the suboptimal response rates for MAPKtargeting therapies [73]. The TP53 gene represents a well-established player in carcinogenesis [73]. Over 50\% of all tumors carry p53 loss-of-function mutations, leading to synthesis of functionally impaired protein products which are unable to transactivate genes that induce cell cycle arrest and apoptosis in response to oncogenic stress [74]. Tumors maintaining wild-type p53 exhibit other types of downstream p53 inactivation, such as hyperactivation of its endogenous post-repressor mouse double minute 2 (MDM2), an ubiquitin ligase 
that catalyzes p53 degradation and inactivation [74]. Most melanomas retain a wild-type p53 and exhibit low TP53 mutation rates, but instead show frequent inactivation of the cyclin-dependent kinase inhibitor 2A (CDKN2A), which eventually lead to MDM2 upregulation and subsequent p53 inhibition [75]. As a result, small-molecule inhibitors that block the p53-MDM2 interaction have been pursued as a new cancer therapeutic strategy for restoring the tumor-suppressive function of p53 in TP53 wild-type tumors [76]. Shattuck-Brandt $e t$ al. [77] recently highlighted the therapeutic efficacy of MDM2 inhibition against TP53WT melanomas with either a wild-type or a mutant BRAF background. They showed that a MDM2 antagonist (namely KRT-232) alone or in combination with BRAF and/or MEK inhibitors can inhibit tumor growth in patient-derived xenografts $(\mathrm{PDX})$ from 15 patients with melanoma by suppressing p53 degradation. MDM2 inhibitor monotherapy was effective against BRAFV600WT tumors, while a combination of KRT-232 plus BRAF/MEK inhibitors exhibited a synergistic effect on BRAFV600E mutant PDXs [77]. In a similar note, our study revealed that a number of small-molecule MDM2 inhibitors show a potential for preventing E2F1-driven metastatic progression.

Overall, our approach predicted AKT1 and MDM2 inhibitors as promising anti-melanoma drugs. If combined with each other or with the standard-of-care regimens for melanoma, such as BRAF and MEK inhibitors, these substances could offer appealing alternative therapeutic strategies, potentially overcoming therapeutic resistance and improving disease-free survival of melanoma patients. Future experiments are essential to confirm the metastasis-preventing potential of such combinations in melanoma animal models. Given that combinations of targeted therapeutics and immunotherapeutics hold a potential to produce durable responses to clinical patients [78], another significant question that is worth further investigation is whether AKT1 and/or MDM2 inhibitors can effectively synergize with checkpoint inhibitors to improve patient survival.

Melanoma is a highly heterogeneous and dynamically evolving cancer type. The increased intratumoral cell diversity can accelerate somatic evolution, because a tumor consisting of a genetically heterogeneous cell population has more possibilities to respond to microenvironmental changes, to evolve, and to spread [79]. It is noteworthy that melanomas can, in several cases, evolve through unorthodox pathways, via the same genes that can successfully inhibit melanoma proliferation. For example, while tyrosinase inhibition is seen as an approach to successfully target proliferative melanoma cells, its loss can trigger EMT-mediated melanoma progression [80]. In a similar manner, stabilization of the tumor-suppressor p53 can drive therapeutic resistance and it was recently suggested that inhibiting rather than activating wild-type p53 may sensitize previously resistant metastatic melanoma cells to therapy [81]. Advanced computational methods, such as artificial intelligence and machine learning are anticipated to shed more light on the roles of target genes and unveil the spatiotemporal complexity of networks underlying metastasis [82] towards developing personalized therapies.

\section{Materials and Methods}

\subsection{Network analysis and motif identification}

The Cytoscape version of the E2F1 map was downloaded from https://sourceforge.net/projects/e2f1map/files and converted into a format suitable for Cytoscape plugin NetDS v3.0 [17]. The purpose of this was to identify important nodes and network motifs in the network. The loop length was set to three nodes and feedback motifs $(n=444)$ were retrieved. We then used the Cytoscape plugin NetworkAnalyzer to evaluate the topological 
properties of nodes [83]. More specifically, we calculated the average number of neighbours for each node in the network (degree) [84] and the density of connections among the neighbours of a node (betweenness centrality) [85] to understand the overall organization of the network. Among non-topological properties, we calculated the number of nodes in a motif involved in the KEGG melanoma pathway (KEGG: 05218), and a prioritization score for each gene from the web resource DISEASES [86] shown in Supplementary file 1.

\subsection{Array data from aggressive melanoma cell lines}

SK-Mel-103 and SK-Mel-147 melanoma cells were harvested 48 hours after infection with Ad.control shRNA or Ad.shE2F1 for RNA extraction. Equal amounts of RNA were analyzed using Affymetrix GeneChip Human Genome U133 Plus 2.0 Arrays (Affymetrix Inc., Santa Clara, CA,). Analysis was done in duplicate for each sample. Background-corrected signal intensities were determined and processed using MAS5 function of the R/Bioconductor affy package (Department of Biostatistical Science, Dana-Farber Cancer Institute, Boston, MA). Gene transcripts not detected in any samples were excluded from statistical analysis. Normalization of expression data, statistical tests, and clustering was accomplished by GeneSpring GX 9.0 (Agilent Technologies Inc, Palo Alto, CA). Expression profiles of both cell lines expressing either control shRNA or shE2F1 were grouped and statistically analyzed using $t$ test and multiple testing correction (Benjamini and Hochberg False Discovery Rate). Only targets displaying a minimum twofold induction or reduction $(P<.05)$ by E2F1 knockdown were included for clustering [3].

\subsection{Motif prioritization}

The regulatory motifs were prioritized using a ranking score for each motifs considering key topological and non-topological properties with respect to the relevance for the melanoma phenotype. The motif ranking score is calculated using Eq. (1).

$$
\begin{aligned}
\text { Ranking score }_{i j} & =\frac{W_{1 j}}{2}\left(\frac{\langle N D\rangle i}{\max \langle N D\rangle}+\frac{\langle B C\rangle i}{\max \langle B C\rangle}\right)+W_{2 j} \frac{\langle D P\rangle i}{\max \langle D P\rangle}+W_{3 j} \frac{\langle G P\rangle i}{\max \langle G P\rangle} \\
& +W_{4 j} \frac{\langle|F C|\rangle i}{\max \langle|F C|\rangle}
\end{aligned}
$$

The equation uses a multi-objective function which is normalized to the maximum property value under consideration. We used a ranking scheme that is previously developed [11] by assigning different weights to various topological and non-topological parameters. In particular, the weights to two topological parameters (node degree $\langle N D\rangle$ and betweenness centrality $\langle B C\rangle$ ) was divided to half for avoiding over-emphasizes topological properties and assigning equal weighting factors $W_{2 j}-W_{4 j}$ to give equal importance to other properties (disease pathway association $\langle D P\rangle$, gene prioritization score $\langle G P\rangle$, Log2 fold change $\langle|F C|\rangle)$ in motif prioritization. The equation generates a ranking score for each motif $i(1 \ldots \mathrm{n})$ depending on the sets of values chosen for the weighting scenarios $j$ (1 to13) shown in Supplementary file 1. Later, top 10 motifs were selected from each of the weighting scenario $(13 \cdot 10=130$ motifs $)$. Furthermore, unique set of motifs were identified and processed for the construction of melanoma-specific core regulatory network. The optimization of multiobjective function is discussed in detail [11].

\subsection{Derivation of core regulatory network}


All the top ranked motif identified in the previous steps were merged to create a regulatory core. Additionally, we also considered receptor proteins as critical factors determining the EMT phenotype and directly interacting/ regulating nodes present in the top-ranked motifs. In total, we found and included ten receptor proteins (AR, ESR1, FGFR1, FLT4, NR2F2, NR4A1, TGFBR1, TGFBR2, THRA, and THRB) into the regulatory core. These receptor proteins are the first neighbors of ranked motif nodes and present in the E2F1 map. In addition, we added four EMT marker proteins (CDH1, VIM, ZEB1, and SNAI1) and direct connections with motif nodes (Supplementary file 1) in our regulatory core.

\subsection{Logic-based modeling to derive protein signatures}

To identify protein signatures in the regulatory core, the network is translated into a logic-based model and in silico perturbation experiments were performed in the software tool CellNetAnalyzer [87]. For this, we derived Boolean rules for the input (receptor proteins) layer and propagation of signals from the input layer to the output layer through the nodes present in the regulatory layer. The network is simulated to determine the impact of the input layer vectors on the EMT phenotype (output layer). We performed single and double perturbation experiments iteratively for the initial conditions that is determined through the additional publicly available gene expression dataset (GSE46517) from Gene Expression Omnibus (GEO). The perturbation experiments were performed by changing the Boolean state of each node alone and in combination to other nodes in the regulatory layer to see the impact on the invasiveness. Those node(s) which upon inhibition change the EMT to minimum level or upon activation to maximum level are further evaluated as effective protein signatures associated with EMT transition in melanoma (Supplementary file 2).

\subsection{Virtual screening of drugs}

For virtual drug screening was performed as follows:

\section{(i) Chemical library preparation}

The lead-like compound subset is downloaded from the ZINC12 database (http://zinc.dock-ing.org/zinc/). Textual descriptors calculated from the 3D SDF files using Open Babel 3.1 .1 (https://pypi.org/project/openbabel/3.1.1/). The compounds are selected meeting Lipinski's rule of five (molecular weight $<500$ Dalton, hydrogen bond donor $<5$, hydrogen bond acceptor $<10$, and an octanol-water partition coefficient iLOGP $<5$ ).

\section{(ii) Structure preparation}

The crystal structure of protein signatures AKT1 (PDB: 3OCB) and MDM2 (PDB: 3JZK) were downloaded from the RCSB Protein Data Bank (https://www.rcsb.org/). Proteins were pre-processed by removal of heteroatoms, adding polar hydrogens, and gasteiger charges using the AutoDock Vina [88]. Further, the coordinates of the active site residues were determined.

\section{(iii) Molecular docking study}

Virtual screening was carried out in PyRx v 0.8 (AutoDock Vina-based) screening tool [89]. The library compounds were first imported as SDF files in open babel of PyRx and further energy minimization of all the library compounds was performed followed by conversion into PDBQT format files. Later, a gird box was designed to cover the binding site residues within the protein signatures and then the library compounds were subjected to docking against AKT1 and MDM2. At each step, the energy of interaction of compound and protein 
was evaluated using binding energy (Kcal/mol) value (Supplementary file 3 ). The docked complexes and graphical visualization were done in DS Visualizer [90].

\section{(iv) ADMET risk and pharmacokinetic prediction}

The docked compounds were sorted based on binding energy ( $\mathrm{kcal} / \mathrm{mol})$ and further filtered by computing a pool of ADMET risk, pharmacokinetics, drug-likeness, and medicinal chemistry friendliness prediction using the SwissADME server (http://www.swissadme.ch/) [57] (Supplementary file 3).

\section{Conclusions}

Cancer is a disease where multiple pathways are dysregulated, and its development and progression involve both independent and overlapping molecular targets. Advanced computational methods can unravel the properties of cancer-related proteins and their interactions in the molecular networks and enable designing of next generation targeted therapeutics. With the computational pipeline used in this study, we were successful in the identification of key protein signatures that derive melanoma metastasis phenotypes using in silico perturbation experiments. Using the virtual screening of lead compounds library, we identified key compounds that bind to AKT1 and MDM2 and suppress their metastatic activity. Some of the top hits were already investigated as potential inhibitors of the identified protein signatures. However, we also find novel hits, some of them were investigated for other caner types and can be further investigated for their potential to check melanoma metastasis. Among the top hits- ZINC000001491367, ZINC000003939645 are predicted as potential inhibitors for AKT1 and ZINC000095605306, ZINC000084689539 for MDM2 inhibition. These compound hits would facilitate the discovery and development of effective inhibitors for clinical use in melanoma metastasis.

\section{Supplementary Materials:}

Supplementary file 1: Four sheets including topological and non-topological properties of three-node FBLs and prioritization score; the top ranked FBLs identified in melanoma; weighting scenarios for motif prioritization; and regulatory core interactions in melanoma.

Supplementary file 2: Effect on EMT phenotype after single/ double perturbation simulation experiments in melanoma.

Supplementary file 3: Two sheets containing binding energies of compounds which bind to protein signatures AKT1 and MDM2; and prediction of ADMET, pharmacokinetic, drug-likeness, and medicinal friendliness score of each docked compound.

Author Contributions: Conceptualization, SG, SL, LB, BP, JV; methodology, SG, NS, JV; software, SG, NS, JV; experiments, SL, NS, FK; writing — original draft preparation, NS, SL, SG, BP; writing — review and editing, OW, SG, JV, BP; visualization, NS, SG, OW, SL, BP; supervision, SG, LB, OW, BP; All authors have read and agreed to the published version of the manuscript.

Funding: This research was funded by the German Federal Ministry of Education and Research (BMBF) projects e:MedMelAutim, grant numbers 01ZX1905A, 01ZX1905B, 01ZX1905D, e:Med-SASKit, grant number 01ZX1903B, Deutsche Krebshilfe, grant number 70112353, and the Deutsche Forschungsgemeinschaft (DFG), grant number PU 188/17-1.

Conflicts of Interest: The authors declare no conflict of interest.

Data Availability Statement: All datasets generated for this study are included in the article/Supplementary Material. 


\section{References}

1. Holderfield, M.; Deuker, M.M.; McCormick, F.; McMahon, M. Targeting RAF kinases for cancer therapy: BRAF-mutated melanoma and beyond. Nature Reviews Cancer 2014, 14, 455-467.

2. Dankort, D.; Curley, D.P.; Cartlidge, R.A.; Nelson, B.; Karnezis, A.N.; Damsky Jr, W.E.; You, M.J.; DePinho, R.A.; McMahon, M.; Bosenberg, M. Braf V600E cooperates with Pten loss to induce metastatic melanoma. Nature genetics 2009, 41, 544-552.

3. Alla, V.; Engelmann, D.; Niemetz, A.; Pahnke, J.; Schmidt, A.; Kunz, M.; Emmrich, S.; Steder, M.; Koczan, D.; Pützer, B.M. E2F1 in melanoma progression and metastasis. Journal of the National Cancer Institute 2010, 102, 127-133.

4. $\quad$ Pützer, B.M.; Steder, M.; Alla, V. Predicting and preventing melanoma invasiveness: advances in clarifying E2F1 function. Expert review of anticancer therapy 2010, 10, 1707-1720.

5. Wang, Y.; Alla, V.; Goody, D.; Gupta, S.K.; Spitschak, A.; Wolkenhauer, O.; Pützer, B.M.; Engelmann, D. Epigenetic factor EPC1 is a master regulator of DNA damage response by interacting with E2F1 to silence death and activate metastasis-related gene signatures. Nucleic acids research 2016, 44, 117-133.

6. Rouaud, F.; Hamouda-Tekaya, N.; Cerezo, M.; Abbe, P.; Zangari, J.; Hofman, V.; Ohanna, M.; Mograbi, B.; El-Hachem, N.; Benfodda, Z. E2F1 inhibition mediates cell death of metastatic melanoma. Cell death \& disease 2018, 9, 1-12.

7. Vera, J.; Schmitz, U.; Lai, X.; Engelmann, D.; Khan, F.M.; Wolkenhauer, O.; Pützer, B.M. Kinetic Modeling-Based Detection of Genetic Signatures That Provide Chemoresistance via the E2F1p73/DNp73-miR-205 Network. Cancer research 2013, 73, 3511-3524.

8. Engelmann, D.; Mayoli-Nüssle, D.; Mayrhofer, C.; Fürst, K.; Alla, V.; Stoll, A.; Spitschak, A.; Abshagen, K.; Vollmar, B.; Ran, S. E2F1 promotes angiogenesis through the VEGF-C/VEGFR-3 axis in a feedback loop for cooperative induction of PDGF-B. Journal of molecular cell biology 2013, 5 , 391-403.

9. Meier, C.; Spitschak, A.; Abshagen, K.; Gupta, S.; Mor, J.M.; Wolkenhauer, O.; Haier, J.; Vollmar, B.; Alla, V.; Pützer, B.M. Association of RHAMM with E2F1 promotes tumour cell extravasation by transcriptional up-regulation of fibronectin. The Journal of pathology 2014, 234, 351-364.

10. Knoll, S.; Fürst, K.; Kowtharapu, B.; Schmitz, U.; Marquardt, S.; Wolkenhauer, O.; Martin, H.; Pützer, B.M. E2F1 induces miR-224/452 expression to drive EMT through TXNIP downregulation. EMBO reports 2014, 15, 1315-1329.

11. Khan, F.M.; Marquardt, S.; Gupta, S.K.; Knoll, S.; Schmitz, U.; Spitschak, A.; Engelmann, D.; Vera, J.; Wolkenhauer, O.; Pützer, B.M. Unraveling a tumor type-specific regulatory core underlying E2F1mediated epithelial-mesenchymal transition to predict receptor protein signatures. Nature communications 2017, 8, 1-15.

12. Logotheti, S.; Marquardt, S.; Gupta, S.K.; Richter, C.; Edelhäuser, B.A.; Engelmann, D.; Brenmoehl, J.; Söhnchen, C.; Murr, N.; Alpers, M. LncRNA-SLC16A1-AS1 induces metabolic reprogramming during Bladder Cancer progression as target and co-activator of E2F1. Theranostics 2020, 10, 9620.

13. Richter, C.; Marquardt, S.; Li, F.; Spitschak, A.; Murr, N.; Edelhäuser, B.A.; Iliakis, G.; Pützer, B.M.; Logotheti, S. Rewiring E2F1 with classical NHEJ via APLF suppression promotes bladder cancer invasiveness. Journal of Experimental \& Clinical Cancer Research 2019, 38, 1-16. 
14. Marquardt, S.; Solanki, M.; Spitschak, A.; Vera, J.; Pützer, B.M. Emerging functional markers for cancer stem cell-based therapies: Understanding signaling networks for targeting metastasis. In Proceedings of the Seminars in cancer biology, 2018; pp. 90-109.

15. Singh, N.; Eberhardt, M.; Wolkenhauer, O.; Vera, J.; Gupta, S.K. An integrative network-driven pipeline for systematic identification of lncRNA-associated regulatory network motifs in metastatic melanoma. BMC bioinformatics 2020, 21, 1-17.

16. Goody, D.; Gupta, S.K.; Engelmann, D.; Spitschak, A.; Marquardt, S.; Mikkat, S.; Meier, C.; Hauser, C.; Gundlach, J.-P.; Egberts, J.-H. Drug repositioning inferred from E2F1-Coregulator interactions studies for the prevention and treatment of metastatic cancers. Theranostics 2019, 9, 1490.

17. Le, D.-H.; Kwon, Y.-K. NetDS: a Cytoscape plugin to analyze the robustness of dynamics and feedforward/feedback loop structures of biological networks. Bioinformatics 2011, 27, 2767-2768.

18. Khan, F.M.; Sadeghi, M.; Gupta, S.K.; Wolkenhauer, O. A network-based integrative workflow to unravel mechanisms underlying disease progression. In Systems Biology; Springer: 2018; pp. 247-276.

19. Glass, D.S.; Jin, X.; Riedel-Kruse, I.H. Nonlinear delay differential equations and their application to modeling biological network motifs. Nature communications 2021, 12, 1-19.

20. Ribas, A.; Flaherty, K.T. BRAF targeted therapy changes the treatment paradigm in melanoma. Nature reviews Clinical oncology 2011, 8, 426-433.

21. Smalley, K.S.; Eroglu, Z.; Sondak, V.K. Combination therapies for melanoma: a new standard of care? American journal of clinical dermatology 2016, 17, 99-105.

22. Curti, B.D.; Faries, M.B. Recent Advances in the Treatment of Melanoma. New England Journal of Medicine 2021, 384, 2229-2240.

23. Ascierto, P.A.; Schadendorf, D.; Berking, C.; Agarwala, S.S.; van Herpen, C.M.; Queirolo, P.; Blank, C.U.; Hauschild, A.; Beck, J.T.; St-Pierre, A. MEK162 for patients with advanced melanoma harbouring NRAS or Val600 BRAF mutations: a non-randomised, open-label phase 2 study. The lancet oncology 2013, 14, 249-256.

24. Logotheti, S.; Pützer, B.M. STAT3 and STAT5 targeting for simultaneous management of melanoma and autoimmune diseases. Cancers 2019, 11, 1448.

25. Holbeck, S.L.; Camalier, R.; Crowell, J.A.; Govindharajulu, J.P.; Hollingshead, M.; Anderson, L.W.; Polley, E.; Rubinstein, L.; Srivastava, A.; Wilsker, D. The National Cancer Institute ALMANAC: a comprehensive screening resource for the detection of anticancer drug pairs with enhanced therapeutic activity. Cancer research 2017, 77, 3564-3576.

26. Julkunen, H.; Cichonska, A.; Gautam, P.; Szedmak, S.; Douat, J.; Pahikkala, T.; Aittokallio, T.; Rousu, J. Leveraging multi-way interactions for systematic prediction of pre-clinical drug combination effects. Nature communications 2020, 11, 1-11.

27. Amirouchene-Angelozzi, N.; Swanton, C.; Bardelli, A. Tumor evolution as a therapeutic target. Cancer discovery 2017, 7, 805-817.

28. Singh, N.; Gupta, S.K. Recent advancement in the early detection of melanoma using computerized tools: An image analysis perspective. Skin Research and Technology 2019, 25, 129-141.

29. Ribeiro, D.M.; Zanzoni, A.; Cipriano, A.; Delli Ponti, R.; Spinelli, L.; Ballarino, M.; Bozzoni, I.; Tartaglia, G.G.; Brun, C. Protein complex scaffolding predicted as a prevalent function of long noncoding RNAs. Nucleic acids research 2018, 46, 917-928. 
30. Singh, N.; Freiesleben, S.; Wolkenhauer, O.; Shukla, Y.; Gupta, S.K. Identification of antineoplastic targets with systems approaches, using resveratrol as an in-depth case study. Current pharmaceutical design 2017, 23, 4773-4793.

31. da Silva Rocha, S.F.; Olanda, C.G.; Fokoue, H.H.; Sant'Anna, C.M. Virtual screening techniques in drug discovery: review and recent applications. Current topics in medicinal chemistry 2019, 19, 17511767.

32. Vera, J.; González-Alcón, C.; Marín-Sanguino, A.; Torres, N. Optimization of biochemical systems through mathematical programming: Methods and applications. Computers \& Operations Research 2010, 37, 1427-1438.

33. Abou-Jaoudé, W.; Traynard, P.; Monteiro, P.T.; Saez-Rodriguez, J.; Helikar, T.; Thieffry, D.; Chaouiya, C. Logical modeling and dynamical analysis of cellular networks. Frontiers in genetics 2016, 7, 94 .

34. Kabbarah, O.; Nogueira, C.; Feng, B.; Nazarian, R.M.; Bosenberg, M.; Wu, M.; Scott, K.L.; Kwong, L.N.; Xiao, Y.; Cordon-Cardo, C. Integrative genome comparison of primary and metastatic melanomas. PloS one 2010, 5, e10770.

35. Lu, X.; Yan, C.; Huang, Y.; Shi, D.; Fu, Z.; Qiu, J.; Yin, Y. Mouse double minute 2 (MDM2) upregulates Snail expression and induces epithelial-to-mesenchymal transition in breast cancer cells in vitro and in vivo. Oncotarget 2016, 7, 37177.

36. Chen, Y.; Wang, D.-D.; Wu, Y.-P.; Su, D.; Zhou, T.-Y.; Gai, R.-H.; Fu, Y.-Y.; Zheng, L.; He, Q.-J.; Zhu, H. MDM2 promotes epithelial-mesenchymal transition and metastasis of ovarian cancer SKOV3 cells. British journal of cancer 2017, 117, 1192-1201.

37. Zhou, Y.; Hu, Y.; Yang, M.; Jat, P.; Li, K.; Lombardo, Y.; Xiong, D.; Coombes, R.; Raguz, S.; Yagüe, E. The miR-106b 25 cluster promotes bypass of doxorubicin-induced senescence and increase in motility and invasion by targeting the E-cadherin transcriptional activator EP300. Cell Death \& Differentiation 2014, 21, 462-474.

38. Liu, B.; Li, X.; Li, C.; Xu, R.; Sun, X. miR-25 mediates metastasis and epithelial-mesenchymaltransition in human esophageal squamous cell carcinoma via regulation of E-cadherin signaling. Bioengineered 2019, 10, 679-688.

39. Wu, J.; Limmer, A.; Narayanan, D.; Doan, H.; Simonette, R.; Rady, P.; Tyring, S. The novel AKT inhibitor Afuresertib suppresses human Merkel cell carcinoma MKL-1 cell growth. Clinical and experimental dermatology $\mathbf{2 0 2 1}$.

40. Kallan, N.C.; Spencer, K.L.; Blake, J.F.; Xu, R.; Heizer, J.; Bencsik, J.R.; Mitchell, I.S.; Gloor, S.L.; Martinson, M.; Risom, T. Discovery and SAR of spirochromane Akt inhibitors. Bioorganic \& medicinal chemistry letters 2011, 21, 2410-2414.

41. Nguyen, T.T.T.; Shingyoji, M.; Hanazono, M.; Zhong, B.; Morinaga, T.; Tada, Y.; Shimada, H.; Hiroshima, K.; Tagawa, M. An MDM2 inhibitor achieves synergistic cytotoxic effects with adenoviruses lacking E1B55kDa gene on mesothelioma with the wild-type p53 through augmenting NFI expression. Cell Death \& Disease 2021, 12, 1-10.

42. $\quad$ Fang, D.D.; Tang, Q.; Kong, Y.; Rong, T.; Wang, Q.; Li, N.; Fang, X.; Gu, J.; Xiong, D.; Yin, Y. MDM2 inhibitor APG-115 exerts potent antitumor activity and synergizes with standard-of-care agents in preclinical acute myeloid leukemia models. Cell death discovery 2021, 7, 1-15.

43. Wang, H.Q.; Mulford, I.J.; Sharp, F.; Liang, J.; Kurtulus, S.; Trabucco, G.; Quinn, D.S.; Longmire, T.A.; Patel, N.; Patil, R. Inhibition of MDM2 Promotes Antitumor Responses in p53 Wild-Type 
Cancer Cells through Their Interaction with the Immune and Stromal Microenvironment. Cancer Research 2021, 81, 3079-3091.

44. Gonzalez-Lopez de Turiso, F.; Sun, D.; Rew, Y.; Bartberger, M.D.; Beck, H.P.; Canon, J.; Chen, A.; Chow, D.; Correll, T.L.; Huang, X. Rational design and binding mode duality of MDM2-p53 inhibitors. Journal of medicinal chemistry 2013, 56, 4053-4070.

45. Freeman-Cook, K.D.; Autry, C.; Borzillo, G.; Gordon, D.; Barbacci-Tobin, E.; Bernardo, V.; Briere, D.; Clark, T.; Corbett, M.; Jakubczak, J. Design of selective, ATP-competitive inhibitors of Akt. Journal of medicinal chemistry 2010, 53, 4615-4622.

46. Seefeld, M.A.; Rouse, M.B.; McNulty, K.C.; Sun, L.; Wang, J.; Yamashita, D.S.; Luengo, J.I.; Zhang, S.; Minthorn, E.A.; Concha, N.O. Discovery of 5-pyrrolopyridinyl-2-thiophenecarboxamides as potent AKT kinase inhibitors. Bioorganic \& medicinal chemistry letters 2009, 19, 2244-2248.

47. Lin, H.; Yamashita, D.S.; Xie, R.; Zeng, J.; Wang, W.; Leber, J.; Safonov, I.G.; Verma, S.; Li, M.; LaFrance, L. Tetrasubstituted pyridines as potent and selective AKT inhibitors: Reduced CYP450 and hERG inhibition of aminopyridines. Bioorganic \& medicinal chemistry letters 2010, 20, 684-688.

48. Wilson, S.C.; Atrash, B.; Barlow, C.; Eccles, S.; Fischer, P.M.; Hayes, A.; Kelland, L.; Jackson, W.; Jarman, M.; Mirza, A. Design, synthesis and biological evaluation of 6-pyridylmethylaminopurines as CDK inhibitors. Bioorganic \& medicinal chemistry 2011, 19, 6949-6965.

49. Wiechmann, S.; Ruprecht, B.; Siekmann, T.; Zheng, R.; Frejno, M.; Kunold, E.; Bajaj, T.; Zolg, D.P.; Sieber, S.A.; Gassen, N.C. Chemical phosphoproteomics sheds new light on the targets and modes of action of AKT inhibitors. ACS Chemical Biology 2021, 16, 631-641.

50. Parthasarathy, S.; Henry, K.; Pei, H.; Clayton, J.; Rempala, M.; Johns, D.; De Frutos, O.; Garcia, P.; Mateos, C.; Pleite, S. Discovery of chiral dihydropyridopyrimidinones as potent, selective and orally bioavailable inhibitors of AKT. Bioorganic \& medicinal chemistry letters 2018, 28, 1887-1891.

51. Chukwuemeka, P.O.; Umar, H.I.; Iwaloye, O.; Oretade, O.M.; Olowosoke, C.B.; Elabiyi, M.O.; Igbe, F.O.; Oretade, O.J.; Eigbe, J.O.; Adeojo, F.J. Targeting p53-MDM2 interactions to identify small molecule inhibitors for cancer therapy: beyond "Failure to rescue". Journal of Biomolecular Structure and Dynamics 2021, 1-19.

52. Lang, L.; Perez, A. Binding Ensembles of p53-MDM2 Peptide Inhibitors by Combining Bayesian Inference and Atomistic Simulations. Molecules 2021, 26, 198.

53. Patil, S.P.; Pacitti, M.F.; Gilroy, K.S.; Ruggiero, J.C.; Griffin, J.D.; Butera, J.J.; Notarfrancesco, J.M.; Tran, S.; Stoddart, J.W. Identification of antipsychotic drug fluspirilene as a potential p53-MDM2 inhibitor: a combined computational and experimental study. Journal of computer-aided molecular design 2015, 29, 155-163.

54. Rew, Y.; Sun, D.; Gonzalez-Lopez De Turiso, F.; Bartberger, M.D.; Beck, H.P.; Canon, J.; Chen, A.; Chow, D.; Deignan, J.; Fox, B.M. Structure-based design of novel inhibitors of the MDM2-p53 interaction. Journal of medicinal chemistry 2012, 55, 4936-4954.

55. Gomha, S.M.; Eldebss, T.M.; Abdulla, M.M.; Mayhoub, A.S. Diphenylpyrroles: novel p53 activators. European journal of medicinal chemistry 2014, 82, 472-479.

56. van Waterschoot, R.A.; Schinkel, A.H. A critical analysis of the interplay between cytochrome P450 3A and P-glycoprotein: recent insights from knockout and transgenic mice. Pharmacological reviews 2011, 63, 390-410.

57. Daina, A.; Michielin, O.; Zoete, V. SwissADME: a free web tool to evaluate pharmacokinetics, druglikeness and medicinal chemistry friendliness of small molecules. Scientific reports 2017, 7, 1-13. 
58. Montanari, F.; Ecker, G.F. Prediction of drug-ABC-transporter interaction-Recent advances and future challenges. Advanced drug delivery reviews 2015, 86, 17-26.

59. Banerjee, P.; Eckert, A.O.; Schrey, A.K.; Preissner, R. ProTox-II: a webserver for the prediction of toxicity of chemicals. Nucleic acids research 2018, 46, W257-W263.

60. Gibbs, J.B. Mechanism-based target identification and drug discovery in cancer research. Science 2000, 287, 1969-1973.

61. Gashaw, I.; Ellinghaus, P.; Sommer, A.; Asadullah, K. What makes a good drug target? Drug discovery today 2011, 16, 1037-1043.

62. Hay, M.; Thomas, D.W.; Craighead, J.L.; Economides, C.; Rosenthal, J. Clinical development success rates for investigational drugs. Nature biotechnology 2014, 32, 40-51.

63. Więckowska, A.; Szałaj, N.; Góral, I.; Bucki, A.; Latacz, G.; Kiec-Kononowicz, K.; Bautista-Aguilera, O.s.M.; Romero, A.; Ramos, E.; Egea, J. In Vitro and In Silico ADME-Tox Profiling and Safety Significance of Multifunctional Monoamine Oxidase Inhibitors Targeting Neurodegenerative Diseases. ACS chemical neuroscience 2020, 11, 3793-3801.

64. Wang, L.; Zhao, H.; Li, J.; Xu, Y.; Lan, Y.; Yin, W.; Liu, X.; Yu, L.; Lin, S.; Du, M.Y. Identifying functions and prognostic biomarkers of network motifs marked by diverse chromatin states in human cell lines. Oncogene 2020, 39, 677-689.

65. Wang, P.; Lü, J.; Yu, X. Identification of important nodes in directed biological networks: A network motif approach. PloS one 2014, 9, e106132.

66. Kitchen, D.B.; Decornez, H.; Furr, J.R.; Bajorath, J. Docking and scoring in virtual screening for drug discovery: methods and applications. Nature reviews Drug discovery 2004, 3, 935-949.

67. Yu, D.H.; Ruan, X.-L.; Huang, J.-Y.; Liu, X.-P.; Ma, H.-L.; Chen, C.; Hu, W.-D.; Li, S. Analysis of the interaction network of hub miRNAs-hub genes, being involved in idiopathic pulmonary fibers and its emerging role in non-small cell lung cancer. Frontiers in genetics 2020, 11, 302.

68. Wei, P.-J.; Wu, F.-X.; Xia, J.; Su, Y.; Wang, J.; Zheng, C.-H. Prioritizing cancer genes based on an improved random walk method. Frontiers in genetics 2020, 11, 377.

69. Cho, J.H.; Robinson, J.P.; Arave, R.A.; Burnett, W.J.; Kircher, D.A.; Chen, G.; Davies, M.A.; Grossmann, A.H.; VanBrocklin, M.W.; McMahon, M. AKT1 activation promotes development of melanoma metastases. Cell reports 2015, 13, 898-905.

70. Irvine, M.; Stewart, A.; Pedersen, B.; Boyd, S.; Kefford, R.; Rizos, H. Oncogenic PI3K/AKT promotes the step-wise evolution of combination BRAF/MEK inhibitor resistance in melanoma. Oncogenesis 2018, 7, 1-11.

71. Shi, H.; Hong, A.; Kong, X.; Koya, R.C.; Song, C.; Moriceau, G.; Hugo, W.; Clarissa, C.Y.; Ng, C.; Chodon, T. A novel AKT1 mutant amplifies an adaptive melanoma response to BRAF inhibition. Cancer discovery 2014, 4, 69-79.

72. Lassen, A.; Atefi, M.; Robert, L.; Wong, D.J.; Cerniglia, M.; Comin-Anduix, B.; Ribas, A. Effects of AKT inhibitor therapy in response and resistance to BRAF inhibition in melanoma. Molecular cancer 2014, 13, 1-14.

73. Lee, J.T.; Herlyn, M. MEK'ing the most of p53 reactivation therapy in melanoma. Journal of Investigative Dermatology 2012, 132, 263-265.

74. Logotheti, S.; Marquardt, S.; Pützer, B.M. p73-governed miRNA networks: translating bioinformatics approaches to therapeutic solutions for cancer metastasis. Computational Biology of Non-Coding RNA

2019, 33-52. 
75. Loureiro, J.B.; Raimundo, L.; Calheiros, J.; Carvalho, C.; Barcherini, V.; Lima, N.R.; Gomes, C.; Almeida, M.I.; Alves, M.G.; Costa, J.L. Targeting p53 for melanoma treatment: counteracting tumour proliferation, dissemination and therapeutic resistance. Cancers 2021, 13, 1648.

76. Beloglazkina, A.; Zyk, N.; Majouga, A.; Beloglazkina, E. Recent small-molecule inhibitors of the p53MDM2 protein-protein interaction. Molecules 2020, 25, 1211.

77. Shattuck-Brandt, R.L.; Chen, S.-C.; Murray, E.; Johnson, C.A.; Crandall, H.; O'Neal, J.F.; Al-Rohil, R.N.; Nebhan, C.A.; Bharti, V.; Dahlman, K.B. Metastatic Melanoma Patient-Derived Xenografts Respond to MDM2 Inhibition as a Single Agent or in Combination with BRAF/MEK Inhibition. Clinical Cancer Research 2020, 26, 3803-3818.

78. Ribas, A.; Algazi, A.; Ascierto, P.A.; Butler, M.O.; Chandra, S.; Gordon, M.; Hernandez-Aya, L.; Lawrence, D.; Lutzky, J.; Miller, W.H. PD-L1 blockade in combination with inhibition of MAPK oncogenic signaling in patients with advanced melanoma. Nature communications 2020, 11, 1-10.

79. McGranahan, N.; Rosenthal, R.; Hiley, C.T.; Rowan, A.J.; Watkins, T.B.; Wilson, G.A.; Birkbak, N.J.; Veeriah, S.; Van Loo, P.; Herrero, J. Allele-specific HLA loss and immune escape in lung cancer evolution. Cell 2017, 171, 1259-1271. e1211.

80. Fuerst, K.; Steder, M.; Logotheti, S.; Angerilli, A.; Spitschak, A.; Marquardt, S.; Schumacher, T.; Engelmann, D.; Herchenroeder, O.; Rupp, R.A. DNp73-induced degradation of tyrosinase links depigmentation with EMT-driven melanoma progression. Cancer letters 2019, 442, 299-309.

81. Webster, M.R.; Fane, M.E.; Alicea, G.M.; Basu, S.; Kossenkov, A.V.; Marino, G.E.; Douglass, S.M.; Kaur, A.; Ecker, B.L.; Gnanapradeepan, K. Paradoxical role for wild-type p53 in driving therapy resistance in melanoma. Molecular cell 2020, 77, 633-644. e635.

82. Fleming, N. Computer-calculated compounds. Nature 2018, 557, S55-57.

83. Assenov, Y.; Ramírez, F.; Schelhorn, S.-E.; Lengauer, T.; Albrecht, M. Computing topological parameters of biological networks. Bioinformatics 2008, 24, 282-284.

84. Wang, X.; Yang, Q.; Liu, M.; Ma, X. Comprehensive influence of topological location and neighbor information on identifying influential nodes in complex networks. Plos one 2021, 16, e0251208.

85. Abbasi, A.; Hossain, L.; Leydesdorff, L. Betweenness centrality as a driver of preferential attachment in the evolution of research collaboration networks. Journal of Informetrics 2012, 6, 403-412.

86. Pletscher-Frankild, S.; Pallejà, A.; Tsafou, K.; Binder, J.X.; Jensen, L.J. DISEASES: Text mining and data integration of disease-gene associations. Methods 2015, 74, 83-89.

87. Klamt, S.; Saez-Rodriguez, J.; Lindquist, J.A.; Simeoni, L.; Gilles, E.D. A methodology for the structural and functional analysis of signaling and regulatory networks. BMC bioinformatics 2006, 7 , $1-26$.

88. Trott, O.; Olson, A.J. AutoDock Vina: improving the speed and accuracy of docking with a new scoring function, efficient optimization, and multithreading. Journal of computational chemistry $\mathbf{2 0 1 0}$, $31,455-461$.

89. Dallakyan, S.; Olson, A.J. Small-molecule library screening by docking with PyRx. In Chemical biology; Springer: 2015; pp. 243-250.

90. Biovia, D.S. Discovery studio modeling environment. 2017. 